\title{
A High Order Adaptive Time-Stepping Strategy and Local Discontinuous Galerkin Method for the Modified Phase Field Crystal Equation
}

\author{
Ruihan $\mathrm{Guo}^{1}$ and Yan $\mathrm{Xu}^{2, *}$ \\ 1 School of Mathematics and Statistics, Zhengzhou University, Zhengzhou, \\ Henan 450001, P.R. China. \\ 2 School of Mathematical Sciences, University of Science and Technology of China, \\ Hefei, Anhui 230026, P.R. China.
}

Received 22 March 2017; Accepted (in revised version) 16 July 2017

\begin{abstract}
In this paper, we will develop a first order and a second order convex splitting, and a first order linear energy stable fully discrete local discontinuous Galerkin (LDG) methods for the modified phase field crystal (MPFC) equation. In which, the first order linear scheme is based on the invariant energy quadratization approach. The MPFC equation is a damped wave equation, and to preserve an energy stability, it is necessary to introduce a pseudo energy, which all increase the difficulty of constructing numerical methods comparing with the phase field crystal (PFC) equation. Due to the severe time step restriction of explicit time marching methods, we introduce the first order and second order semi-implicit schemes, which are proved to be unconditionally energy stable. In order to improve the temporal accuracy, the semi-implicit spectral deferred correction (SDC) method combining with the first order convex splitting scheme is employed. Numerical simulations of the MPFC equation always need long time to reach steady state, and then adaptive time-stepping method is necessary and of paramount importance. The schemes at the implicit time level are linear or nonlinear and we solve them by multigrid solver. Numerical experiments of the accuracy and long time simulations are presented demonstrating the capability and efficiency of the proposed methods, and the effectiveness of the adaptive time-stepping strategy.
\end{abstract}

AMS subject classifications: 65M60, 35L75, 35G25

Key words: Adaptive time-stepping, local discontinuous Galerkin method, modified phase field crystal equation, convex splitting, pseudo energy, unconditionally energy stable, spectral deferred correction.

\section{Introduction}

In this paper, we consider local discontinuous Galerkin (LDG) spatial discretization and high order semi-implicit time marching methods in a bounded domain $\Omega \in \mathbb{R}^{d}(d \leq 2)$ for

*Corresponding author. Email addresses: rguo@zzu. edu.cn (R. Guo), yxu@ustc. edu.cn (Y. Xu) 
the modified phase field crystal (MPFC) equation:

$$
\partial_{t t} \phi+\beta \partial_{t} \phi=\nabla \cdot\left(M(\phi) \nabla\left(\phi^{3}+(1-\epsilon) \phi+2 \Delta \phi+\Delta^{2} \phi\right)\right),
$$

where $M(\phi) \geq 0$ is a mobility, $\beta>0$ and $\epsilon \leq 1$.

In equation (1.1), when $\beta=0$, it is known as the phase field crystal (PFC) equation. The PFC model was recently proposed by Elder et al. $[10,11]$ to study the nonequilibrium microstructure formation by introducing a free energy functional of the local-time-averaged density field. There have been many algorithms developed and simulations performed for the PFC equation recently, using finite difference methods $[17,23,27]$, finite element methods [13] and local discontinuous Galerkin method [15]. The MPFC equation is an important extension of the PFC equation, and it was introduced in [20,21]. The MPFC equation (1.1) is a nonlinear damped wave equation, which models a viscoelastic response to perturbations to the density field. The PFC and MPFC equations have close relationship, and it is possible to apply numerical methods for the former equation to the latter one. However, one should keep in mind that the original energy of the MPFC equation may increase in time on some time intervals, so it is desirable to introduce a pseudo energy. Thus, we have to be very careful when designing energy stable convex splitting schemes for the MPFC equation.

There are a few numerical methods for the simulations of the MPFC equation. For example, Wang et al. [1,22] developed energy stable and convergent finite difference schemes for the MPFC equation. Baskaran et al. [2] provided a detailed convergence analysis for an unconditionally energy stable, second-order accurate convex splitting scheme for the MPFC equation. In which, the spatial discretization was central finite difference method, and only second order spatial accuracy was achieved. Grasselli and Pierre [14] proposed an energy stable and convergence finite element schemes for the MPFC equation. In this paper, an LDG spatial discretization method will be developed, obtaining arbitrary high order accuracy by choosing the local approximating basis. For temporal discretization, we will first construct the first order and second order convex splitting schemes, the first order linear scheme, and then employ the semi-implicit spectral deferred correction (SDC) method [24] to achieve high order temporal accuracy. To the best of our knowledge, the first order linear scheme and the second order convex splitting scheme are novel combined with the pseudo energy that we define.

The discontinuous Galerkin (DG) method is a class of finite element methods, using completely discontinuous, piecewise polynomials as the solution and the test spaces. It was first designed as a method for solving hyperbolic conservation laws containing only first order spatial derivatives, e.g. Reed and Hill [18] for solving linear equations, and later Cockburn et al. [5-8] for solving nonlinear equations.

It is not straightforward to apply the DG method directly to PDEs containing higher order spatial derivatives, therefore the LDG method was introduced. There are usually two steps to implement the LDG method: first rewriting the problem into a form which contains only first order derivatives, and then applying the DG method by choosing appropriate numerical fluxes to ensure stability. The first LDG method was constructed 
by Cockburn and Shu [9] for solving time dependent convection diffusion systems, motivated by the work of Bassi and Rebay [3] for compressible Navier-Stokes equations. Following these pioneering papers, LDG methods have been successfully designed and applied in a number of models involving diffusion and dispersive problems (see for example the review paper [25]). DG and LDG methods also have several attractive properties, for example: allowing for efficient $h, p$ adaptivity and having excellent parallel efficiency.

The MPFC equation is a sixth-order nonlinear damped wave equation, and explicit time marching methods require extremely small time step to maintain stability. Therefore, it would be desirable to introduce implicit or semi-implicit time discretization techniques to alleviate this problem. In this paper, we will develop the first order and second order semi-implicit schemes for the MPFC equation and prove the corresponding unconditional energy stability. However, the schemes are only first order or second order accurate in time. To improve the temporal accuracy, we employ the semi-implicit SDC method [24], combining with the proposed first order convex splitting scheme.

The MPFC equation itself always experience long time evolution therefore computational efficiency is essential to map out the whole dynamics from initial state to steady state. The proposed semi-implicit time marching methods allow larger time step and indeed improve the efficiency. To further improve the efficiency of our schemes, we will employ the adaptive time-stepping strategy. We know that the energy is an important quantity in the MPFC equation and here the adaptive time steps are determined based on the time derivative of the energy. In which, when the energy decays rapidly, small time steps are adopted, and when the energy decays slowly, large time steps will be used. Zhang et al. [27] employed this idea for solving the PFC equation and showed that the adaptive time-stepping technique was efficient numerically.

The rest of the paper is organized as follows. In Section 2, we give a brief review on the properties of the MPFC equation and present the first order and second order schemes. In Section 3, we develop LDG methods for the proposed schemes, and prove the corresponding fully discrete schemes are unconditionally energy stable. In Section 4, we combine the semi-implicit SDC method with the first order convex splitting scheme to obtain high order temporal accuracy. We also give a simple description of the adaptive time-stepping strategy. Section 5 contains numerical results, demonstrating the accuracy and effectiveness of the proposed numerical methods. Finally, we give concluding remarks in Section 6.

\section{The MPFC equation and proposed semi-implicit temporal schemes}

\subsection{The MPFC equation}

We consider a dimensionless energy of the form 


$$
E(\phi)=\int_{\Omega}\left\{\frac{1}{4} \phi^{4}+\frac{1-\epsilon}{2} \phi^{2}-|\nabla \phi|^{2}+\frac{1}{2}(\Delta \phi)^{2}\right\},
$$

where $\Omega \in \mathbb{R}^{d}, \phi: \Omega \rightarrow \mathbb{R}$ is the atomic density field, and $\epsilon$ is a constant. We assume here that $\Omega=\left(0, L_{x}\right) \times\left(0, L_{y}\right)$ and that $\phi$ and $\Delta \phi$ are periodic on $\partial \Omega$ for simplicity. Then the MPFC equation is the pseudo-gradient flow

$$
\partial_{t t} \phi+\beta \partial_{t} \phi=\nabla \cdot(M(\phi) \nabla \mu),
$$

where $\mu$ is the chemical potential with respect to $E$ and takes the following form

$$
\mu:=\frac{\delta E}{\delta \phi}=\phi^{3}+(1-\epsilon) \phi+2 \Delta \phi+\Delta^{2} \phi .
$$

For simplicity, we take $M$ to be a constant and consider the simpler equation

$$
\partial_{t t} \phi+\beta \partial_{t} \phi=M \Delta \mu, \quad \mu=\phi^{3}+(1-\epsilon) \phi+2 \Delta \phi+\Delta^{2} \phi .
$$

The presence of the term $\partial_{t t} \phi$ is a nontrivial modification from the mathematical point of view. Indeed, different from the sixth order parabolic type PFC equation, the MPFC equation (2.4) is not a gradient equation, and the energy (2.1) may actually increase in time on some time intervals, we will show the phenomenon numerically in Section 5. However, solutions of the MPFC equation do dissipate a pseudo energy. Therefore, in order to design numerical schemes that preserve an energy stability, it is necessary and of great importance to introduce an appropriate pseudo energy.

To introduce the pseudo energy, and activated by the idea in [12], we first recast the MPFC equation (2.4) as the following system of equations

$$
\left\{\begin{array}{l}
\phi_{t}=\Delta u, \\
\frac{\partial \Delta u}{\partial t}=M \Delta \mu-\beta \Delta u .
\end{array}\right.
$$

Then we define the pseudo energy

$$
\mathcal{E}(\phi, u):=E(\phi)+\frac{1}{2 M} \int_{\Omega}|\nabla u|^{2} d x .
$$

The pseudo energy is non-increasing with respect to time $t$, i.e.

$$
\begin{aligned}
\frac{d}{d t} \mathcal{E}(\phi, u) & =\int_{\Omega}\left\{\phi^{3} \phi_{t}+(1-\epsilon) \phi \phi_{t}-2 \nabla \phi \cdot \nabla \phi_{t}+\Delta \phi \Delta \phi_{t}+\frac{1}{M} \nabla u \cdot \nabla u_{t}\right\} d x \\
& =\int_{\Omega}\left\{\mu \phi_{t}-\frac{1}{M} \Delta u_{t} u\right\} d x \\
& =\int_{\Omega}\left\{\mu \Delta u-\frac{1}{M}(\Delta \mu-\beta \Delta u) u\right\} d x \\
& =-\frac{\beta}{M} \int_{\Omega} \nabla u \cdot \nabla u d x \\
& \leq 0 .
\end{aligned}
$$


Then, we will devote to constructing numerical schemes that preserve the energy stability.

\subsection{The discrete time, continuous space schemes}

The numerical simulations of the MPFC equation always need long time to reach steady state. And because of the high stiffness of the MPFC equation, explicit time discretization methods will suffer from extremely small time step restriction $\left(\Delta t=\mathcal{O}\left(\Delta x^{6}\right)\right)$ for stability, but not for accuracy. Therefore, it would be desirable to develop implicit time marching methods to alleviate this problem. Here we describe semi-implicit time-discrete schemes for the MPFC equation (2.5). The first and second order convex splitting schemes are based on convex splitting approaches that have been widely used for phase field problems. The first order linear scheme is based on the invariant energy quadratization approach.

\section{First order convex splitting scheme}

In order to develop the convex splitting scheme, we first split the energy (2.1) as $E(\phi)=$ $E_{c}(\phi)-E_{e}(\phi)$, where $E_{c}(\phi)$ and $E_{e}(\phi)$ represent the contractive term and the expansive term, respectively, and are given as

$$
E_{c}(\phi)=\int_{\Omega}\left\{\frac{1}{2}(\Delta \phi)^{2}+\frac{1}{4} \phi^{4}+\frac{1-\epsilon}{2} \phi^{2}\right\} d x, \quad E_{e}(\phi)=\int_{\Omega}\left\{|\nabla \phi|^{2}\right\} d x .
$$

By treating the contractive term $E_{c}(\phi)$ implicitly and the expansive term $E_{e}(\phi)$ explicitly, we get the convex splitting scheme as follows

$$
\left\{\begin{array}{l}
\frac{\phi^{n+1}-\phi^{n}}{\Delta t}=\Delta u^{n+1}, \\
\frac{\Delta u^{n+1}-\Delta u^{n}}{\Delta t}=M \Delta \mu^{n+1}-\beta \Delta u^{n+1} \\
\mu^{n+1}=\left(\phi^{n+1}\right)^{3}+(1-\epsilon) \phi^{n+1}+2 \Delta \phi^{n}+\Delta^{2} \phi^{n+1} .
\end{array}\right.
$$

\section{Second order convex splitting scheme}

Activated by the work in [1], we develop a novel second order convex splitting scheme for the MPFC equation (2.5) as follows:

$$
\left\{\begin{array}{l}
\frac{\phi^{n+1}-\phi^{n}}{\Delta t}=\Delta u^{n+\frac{1}{2}}, \\
\frac{\Delta u^{n+1}-\Delta u^{n}}{\Delta t}=M \Delta \mu^{n+1}-\beta \Delta u^{n+\frac{1}{2}}, \\
\mu^{n+1}=(1-\epsilon) \phi^{n+\frac{1}{2}}+\frac{\left(\phi^{n+1}\right)^{2}+\left(\phi^{n}\right)^{2}}{2} \phi^{n+\frac{1}{2}}+3 \Delta \phi^{n}-\Delta \phi^{n-1}+\Delta^{2} \phi^{n+\frac{1}{2}},
\end{array}\right.
$$


where $\phi^{-1}:=\phi^{0}$, and

$$
u^{n+\frac{1}{2}}=\frac{1}{2}\left(u^{n+1}+u^{n}\right), \quad \phi^{n+\frac{1}{2}}=\frac{1}{2}\left(\phi^{n+1}+\phi^{n}\right) .
$$

\section{First order linear scheme}

In the following, we will prove the unconditional energy stability of the convex splitting schemes (2.8) and (2.9). But as for the unique solvability, the proofs are much more elusive in the LDG framework. Therefore, we will then develop a linearly first order, unconditionally energy stable scheme for the MPFC equation inspired by the invariant energy quadratization approach [26] to discrete the nonlinear term.

In order to develop the linear scheme, we first introduce an auxiliary function as follows

$$
U=\phi^{2}
$$

We recast the energy functional (2.1) as

$$
E(\phi, U)=\int_{\Omega}\left\{\frac{1}{4} U^{2}+\frac{1-\epsilon}{2} \phi^{2}-|\nabla \phi|^{2}+\frac{1}{2}(\Delta \phi)^{2}\right\},
$$

and the equivalent equation is given as

$$
\left\{\begin{array}{l}
\phi_{t}=\Delta u, \\
\frac{\partial \Delta u}{\partial t}=M \Delta \mu-\beta \Delta u, \\
\mu=U \phi+(1-\epsilon) \phi+2 \Delta \phi+\Delta^{2} \phi,
\end{array}\right.
$$

where $U$ satisfies $U_{t}=2 \phi \phi_{t}$. Then the corresponding first order linear scheme is

$$
\left\{\begin{array}{l}
\frac{\phi^{n+1}-\phi^{n}}{\Delta t}=\Delta u^{n+1}, \\
\frac{\Delta u^{n+1}-\Delta u^{n}}{\Delta t}=M \Delta \mu^{n+1}-\beta \Delta u^{n+1}, \\
\mu^{n+1}=U^{n+1} \phi^{n}+(1-\epsilon) \phi^{n+1}+2 \Delta \phi^{n}+\Delta^{2} \phi^{n+1}, \\
\frac{U^{n+1}-U^{n}}{\Delta t}=2 \phi^{n} \frac{\phi^{n+1}-\phi^{n}}{\Delta t} .
\end{array}\right.
$$

\section{Fully discrete energy stable local discontinuous Galerkin schemes}

In this section, we will develop local discontinuous Galerkin (LDG) spatial discretization methods for the first order convex splitting scheme (2.8), the second order scheme (2.9) and the first order linear scheme (2.12), to obtain fully discrete stable LDG schemes for the MPFC equation, and prove the corresponding unconditional energy stabilities. 


\subsection{Notations}

We consider a subdivision $\mathcal{T}_{h}$ of $\Omega$ with shape-regular elements $K$. Let $\mathcal{E}_{h}$ denote the union of the boundary faces of elements $K \in \mathcal{T}_{h}$, i.e. $\mathcal{E}_{h}=\bigcup_{K \in \mathcal{T}_{h}} \partial K$, and $\mathcal{E}_{0}=\mathcal{E}_{h} \backslash \partial \Omega$. Let $\mathcal{P}^{k}(K)$ be the space of polynomials of degree at most $k \geq 0$ on $K \in \mathcal{T}_{h}$. The discontinuous Galerkin finite element spaces are denoted by

$$
\begin{aligned}
& V_{h}=\left\{\varphi:\left.\varphi\right|_{K} \in \mathcal{P}^{k}(K), \forall K \in \mathcal{T}_{h}\right\}, \\
& \Sigma_{h}^{d}=\left\{\Phi=\left(\phi_{1}, \cdots, \phi_{d}\right)^{T}:\left.\phi_{l}\right|_{K} \in \mathcal{P}^{k}(K), l=1, \cdots, d, \forall K \in \mathcal{T}_{h}\right\} .
\end{aligned}
$$

Notice that functions in $V_{h}$ and $\Sigma_{h}^{d}$ are allowed to be completely discontinuous across element interfaces.

In order to describe the flux functions, we need to introduce some notations. Let $e$ be an interior face shared by the "left" and "right" elements $K_{L}$ and $K_{R}$ and define the normal vectors $v_{L}$ and $v_{R}$ on $e$ pointing exterior to $K_{L}$ and $K_{R}$, respectively. For our purpose, "left" and "right" can be uniquely defined for each face according to any fixed rule. For example, we choose $v_{0}$ as a constant vector. The left element $K_{L}$ to the face $e$ requires that $\boldsymbol{v}_{L} \cdot \boldsymbol{v}_{0}<0$, and the right one $K_{R}$ requires $v_{L} \cdot v_{0} \geq 0$. If $\psi$ is a function on $K_{L}$ and $K_{R}$, but possibly discontinuous across $e$, let $\psi_{L}$ denote $\left.\left(\left.\psi\right|_{K_{L}}\right)\right|_{e}$ and $\psi_{R}$ denote $\left.\left(\left.\psi\right|_{K_{R}}\right)\right|_{e}$, the left and right trace, respectively.

\subsection{First order fully discrete LDG scheme}

To construct the LDG method for the first order convex splitting scheme (2.8), we first rewrite it as a first order system:

$$
\begin{aligned}
\frac{\phi^{n+1}-\phi^{n}}{\Delta t} & =\nabla \cdot s^{n+1}, \\
s^{n+1} & =\nabla u^{n+1}, \\
\frac{\nabla \cdot s^{n+1}-\nabla \cdot s^{n}}{\Delta t} & =M \nabla \cdot p^{n+1}-\beta \nabla \cdot s^{n+1}, \\
\boldsymbol{p}^{n+1} & =\nabla\left(r^{n+1}+2 q^{n}+q_{2}^{n+1}\right), \\
q_{2}^{n+1} & =\nabla \cdot \boldsymbol{q}_{1}^{n+1}, \\
\boldsymbol{q}_{1}^{n+1} & =\nabla q^{n+1}, \\
q^{n+1} & =\nabla \cdot \boldsymbol{w}^{n+1}, \\
\boldsymbol{w}^{n+1} & =\nabla \phi^{n+1}, \\
r^{n+1} & =\left(\phi^{n+1}\right)^{3}+(1-\epsilon) \phi^{n+1} .
\end{aligned}
$$

To simplify the notation, we still use $\phi^{n+1}, u^{n+1}, q_{2}^{n+1}, q^{n+1}, r^{n+1}, s^{n+1}, p^{n+1}, q_{1}^{n+1}, w^{n+1}$ as the numerical solution. The LDG scheme to solve the system (3.1) becomes the following: Find $\phi^{n+1}, u^{n+1}, q_{2}^{n+1}, q^{n+1}, r^{n+1} \in V_{h}$, and $s^{n+1}, p^{n+1}, q_{1}^{n+1}, w^{n+1} \in \Sigma_{h}^{d}$, such that, for all 
test functions $\varphi_{1}, \varphi_{2}, \varphi_{3}, \varphi_{4}, \varphi_{5} \in V_{h}$ and $\boldsymbol{\theta}_{1}, \boldsymbol{\theta}_{2}, \boldsymbol{\theta}_{3}, \boldsymbol{\theta}_{4} \in \Sigma_{h}^{d}$, we have

$$
\begin{aligned}
\int_{K} \frac{\phi^{n+1}-\phi^{n}}{\Delta t} \varphi_{1} d K= & -\int_{K} s^{n+1} \cdot \nabla \varphi_{1} d K+\int_{\partial K} \widehat{\boldsymbol{s}}^{n+1} \cdot \boldsymbol{v} \varphi_{1} d s \\
\int_{K} s^{n+1} \cdot \boldsymbol{\theta}_{1} d K= & -\int_{K} u^{n+1} \nabla \cdot \boldsymbol{\theta}_{1} d K+\int_{\partial K} \widehat{u}^{n+1} \boldsymbol{\theta}_{1} \cdot \boldsymbol{v} d s \\
& =\int_{K} \frac{\boldsymbol{s}^{n+1}-\boldsymbol{s}^{n}}{\Delta t} \cdot \nabla \varphi_{2} d K-\int_{\partial K} \frac{\left(\widehat{\boldsymbol{s}}^{n+1}-\widehat{\boldsymbol{s}}^{n}\right) \cdot \boldsymbol{v}}{\Delta t} \varphi_{2} d s \\
& -\int_{K}\left(M \boldsymbol{p}^{n+1}-\beta s^{n+1}\right) \cdot \nabla \varphi_{2} d K+\int_{\partial K}\left(M \widehat{\boldsymbol{p}}^{n+1}-\beta \widehat{\boldsymbol{s}}^{n+1}\right) \cdot \boldsymbol{v} \varphi_{2} d s \\
\int_{K} \boldsymbol{p}^{n+1} \cdot \boldsymbol{\theta}_{2} d K= & -\int_{K}\left(r^{n+1}+2 q^{n}+q_{2}^{n+1}\right) \nabla \cdot \boldsymbol{\theta}_{2} d K+\int_{\partial K}\left(\widehat{r}^{n+1}+2 \widehat{q}^{n}+\widehat{q}_{2}^{n+1}\right) \boldsymbol{\theta}_{2} \cdot \boldsymbol{v} d s \\
\int_{K} q^{n+1} \varphi_{3} d K= & -\int_{K} \boldsymbol{q}_{1}^{n+1} \cdot \nabla \varphi_{3} d K+\int_{\partial K} \widehat{\boldsymbol{q}}_{1}^{n+1} \cdot \boldsymbol{v} \varphi_{3} d K= \\
\int_{K} q^{n+1} \varphi_{4} d K= & -\int_{K} q^{n+1} \nabla \cdot \int_{K} \boldsymbol{\theta}^{n+1} \cdot \nabla K+\int_{\partial K} \widehat{q}^{n+1} \boldsymbol{\theta}_{3} \cdot \boldsymbol{v} d s \\
\int_{K} \boldsymbol{w}^{n+1} \cdot \boldsymbol{\theta}_{4} d K & =-\int_{K} \phi^{n+1} \nabla \cdot \int_{\partial K} \widehat{\boldsymbol{w}}^{n+1} \cdot \boldsymbol{v} \varphi_{4} d S+\int_{\partial K} \widehat{\phi}^{n+1} \boldsymbol{\theta}_{4} \cdot \boldsymbol{v} d s \\
\int_{K} r^{n+1} \varphi_{5} d K & =\int_{K}\left(\left(\phi^{n+1}\right)^{3}+(1-\epsilon) \phi^{n+1}\right) \varphi_{5} d K
\end{aligned}
$$

The "hat" terms in scheme (3.2) in the cell boundary terms from integration by parts are the so-called "numerical fluxes", which are functions defined on the edges and should be designed based on different guiding principles for different PDEs to ensure stability and local solvability of the intermediate variables. It turns out that we can take the simple choices such as

$$
\begin{aligned}
& \widehat{\boldsymbol{p}}^{n+1}=\boldsymbol{p}_{L}^{n+1}, \widehat{u}^{n+1}=u_{R}^{n+1}, \widehat{\boldsymbol{s}}^{n+1}=\boldsymbol{s}_{L}^{n+1}, \widehat{q}_{2}^{n+1}=q_{2 R}^{n+1}, \widehat{r}^{n+1}=r_{R}^{n+1}, \\
& \widehat{\boldsymbol{q}}_{1}^{n+1}=\boldsymbol{q}_{1 L}^{n+1}, \widehat{q}^{n+1}=q_{R}^{n+1}, \widehat{\boldsymbol{w}}^{n+1}=\boldsymbol{w}_{L}^{n+1}, \widehat{\phi}^{n+1}=\phi_{R}^{n+1} .
\end{aligned}
$$

We remark that the choice for the fluxes (3.3) is not unique. Considering the compactness of the stencil and the optimal accuracy, the crucial part is taking $\widehat{\boldsymbol{s}}^{n+1}, \widehat{\boldsymbol{p}}^{n+1}$ and $\widehat{u}^{n+1}$ from opposite sides, $\widehat{\boldsymbol{s}}^{n+1}$ and $\widehat{r}^{n+1}, \widehat{q}^{n+1}, \widehat{q}_{2}^{n+1}$ from opposite sides, $\widehat{\boldsymbol{q}}_{1}^{n+1}, \widehat{\boldsymbol{w}}^{n+1}$ and $\widehat{\phi}^{n+1}$ from opposite sides, and $\widehat{q}^{n+1}$ and $\widehat{w}^{n+1}$ from opposite sides.

It is easy to see that the first order fully discrete LDG scheme (3.2) is mass conservative, by choosing the test function $\varphi_{1}=1$ in scheme (3.2).

Proposition 3.1. The solution to the LDG scheme (3.2) with the periodic boundary condition satisfies the mass conservation

$$
\int_{\Omega} \phi^{n+1} d x=\int_{\Omega} \phi^{n} d x
$$


Next, we will prove the energy stability for the fully discrete LDG scheme (3.2) with the choice of the numerical fluxes (3.3).

Proposition 3.2. (Energy stability for the first order fully discrete LDG scheme). The solution to the LDG scheme (3.2) with the numerical fluxes (3.3) and the periodic boundary condition satisfies the energy stability

$$
\mathcal{E}_{h}\left(q^{n+1}, \boldsymbol{w}^{n+1}, \boldsymbol{s}^{n+1}, \phi^{n+1}\right)-\mathcal{E}_{h}\left(q^{n}, \boldsymbol{w}^{n}, \boldsymbol{s}^{n}, \phi^{n}\right) \leq 0,
$$

where

$$
\mathcal{E}_{h}(q, w, s, \phi)=\int_{\Omega}\left(\frac{1}{2} q^{2}-w \cdot w+\frac{1}{2 M} s \cdot s+\frac{1}{4} \phi^{4}+\frac{1-\epsilon}{2} \phi^{2}\right) d x .
$$

Proof. Let $\mathcal{D} \phi$ denote $\phi^{n+1}-\phi^{n}$. For Eqs. (3.2g) and (3.2h) of the LDG scheme, subtracting the equations at time level $t^{n}$ from the equations at time level $t^{n+1}$, respectively, we get

$$
\begin{aligned}
\int_{K} \mathcal{D} q \varphi_{4} d K & =-\int_{K} \mathcal{D} \boldsymbol{w} \cdot \nabla \varphi_{4} d K+\int_{\partial K} \mathcal{D} \widehat{\boldsymbol{w}} \cdot \boldsymbol{v} \varphi_{4} d s, \\
\int_{K} \mathcal{D} \boldsymbol{w} \cdot \boldsymbol{\theta}_{4} d K & =-\int_{K} \mathcal{D} \phi \nabla \cdot \boldsymbol{\theta}_{4} d K+\int_{\partial K} \mathcal{D} \widehat{\phi} \boldsymbol{\theta}_{4} \cdot \boldsymbol{v} d s
\end{aligned}
$$

where $\mathcal{D} q=q^{n+1}-q^{n}$ and $\mathcal{D} w=w^{n+1}-w^{n}$. Then taking the test functions

$$
\varphi_{4}=\frac{1}{\Delta t} q^{n+1}, \quad \boldsymbol{\theta}_{4}=-\frac{1}{\Delta t} q_{1}^{n+1}-\frac{2}{\Delta t} w^{n}
$$

we obtain

$$
\begin{aligned}
\frac{1}{\Delta t} \int_{K} \mathcal{D} q q^{n+1} d K= & -\frac{1}{\Delta t} \int_{K} \mathcal{D} \boldsymbol{w} \cdot \nabla q^{n+1} d K+\frac{1}{\Delta t} \int_{\partial K} \mathcal{D} \widehat{\boldsymbol{w}} \cdot \boldsymbol{v} q^{n+1} d s, \\
-\frac{1}{\Delta t} \int_{K} \mathcal{D} \boldsymbol{w} \cdot\left(\boldsymbol{q}_{1}^{n+1}+2 \boldsymbol{w}^{n}\right) d K= & \frac{1}{\Delta t} \int_{K} \mathcal{D} \phi \nabla \cdot\left(\boldsymbol{q}_{1}^{n+1}+2 \boldsymbol{w}^{n}\right) d K \\
& -\frac{1}{\Delta t} \int_{\partial K} \mathcal{D} \widehat{\phi}\left(\boldsymbol{q}_{1}^{n+1}+2 \boldsymbol{w}^{n}\right) \cdot \boldsymbol{v} d s .
\end{aligned}
$$

For Eq. (3.2g), choosing the test function $\varphi_{4}=-\frac{2}{\Delta t} \mathcal{D} \phi$ at time level $t^{n}$ leads to

$$
-\frac{2}{\Delta t} \int_{K} q^{n} \mathcal{D} \phi d K=\frac{2}{\Delta t} \int_{K} w^{n} \cdot \nabla \mathcal{D} \phi d K-\frac{2}{\Delta t} \int_{\partial K} \widehat{\boldsymbol{w}}^{n} \cdot v \mathcal{D} \phi d s .
$$

For other equations in scheme (3.2), we choose the test functions

$$
\begin{aligned}
& \varphi_{1}=r^{n+1}+2 q^{n}+q_{2}^{n+1}, \theta_{1}=-p^{n+1}+\frac{\beta}{M} s^{n+1}+\frac{1}{M \Delta t} \mathcal{D} s, \varphi_{2}=-\frac{1}{M} u^{n+1}, \\
& \theta_{2}=s^{n+1}, \varphi_{3}=-\frac{1}{\Delta t} \mathcal{D} \phi, \theta_{3}=\frac{1}{\Delta t} \mathcal{D} \boldsymbol{w}, \varphi_{5}=-\frac{1}{\Delta t} \mathcal{D} \phi,
\end{aligned}
$$


respectively, to get

$$
\begin{aligned}
& \frac{1}{\Delta t} \int_{K} \mathcal{D} \phi\left(r^{n+1}+2 q^{n}+q_{2}^{n+1}\right) d K=-\int_{K} s^{n+1} \cdot \nabla\left(r^{n+1}+2 q^{n}+q_{2}^{n+1}\right) d K \\
& +\int_{\partial K} \widehat{\boldsymbol{s}}^{n+1} \cdot \boldsymbol{v}\left(r^{n+1}+2 q^{n}+q_{2}^{n+1}\right) d s, \\
& \int_{K} s^{n+1} \cdot\left(\frac{1}{M \Delta t} \mathcal{D} s-p^{n+1}+\frac{\beta}{M} s^{n+1}\right) d K=-\int_{K} u^{n+1} \nabla \cdot\left(\frac{1}{M \Delta t} \mathcal{D} s-p^{n+1}+\frac{\beta}{M} s^{n+1}\right) d K \\
& +\int_{\partial K} \widehat{u}^{n+1}\left(\frac{1}{M \Delta t} \mathcal{D} s-p^{n+1}+\frac{\beta}{M} s^{n+1}\right) \cdot v d s, \\
& 0=-\int_{K} \frac{s^{n+1}-s^{n}}{M \Delta t} \cdot \nabla u^{n+1} d K+\int_{\partial K} \frac{\widehat{\boldsymbol{s}}^{n+1} \cdot \boldsymbol{v}-\widehat{\boldsymbol{s}}^{n} \cdot \boldsymbol{v}}{M \Delta t} u^{n+1} d s \\
& +\int_{K}\left(p^{n+1}-\frac{\beta}{M} s^{n+1}\right) \cdot \nabla u^{n+1} d K-\int_{\partial K}\left(\widehat{\boldsymbol{p}}^{n+1} \cdot \boldsymbol{v}-\frac{\beta}{M} \widehat{\boldsymbol{s}}^{n+1} \cdot \boldsymbol{v}\right) u^{n+1} d s, \\
& \int_{K} p^{n+1} \cdot s^{n+1} d K=-\int_{K}\left(r^{n+1}+2 q^{n}+q_{2}^{n+1}\right) \nabla \cdot s^{n+1} d K \\
& +\int_{\partial K}\left(\widehat{r}^{n+1}+2 \widehat{q}^{n}+\widehat{q}_{2}^{n+1}\right) s^{n+1} \cdot v d s, \\
& -\frac{1}{\Delta t} \int_{K} q_{2}^{n+1} \mathcal{D} \phi d K=\frac{1}{\Delta t} \int_{K} q_{1}^{n+1} \cdot \nabla \mathcal{D} \phi d K-\frac{1}{\Delta t} \int_{\partial K} \widehat{\boldsymbol{q}}_{1}^{n+1} \cdot \boldsymbol{v} \mathcal{D} \phi d s, \\
& \frac{1}{\Delta t} \int_{K} \boldsymbol{q}_{1}^{n+1} \cdot \mathcal{D} w d K=-\frac{1}{\Delta t} \int_{K} q^{n+1} \nabla \cdot \mathcal{D} w d K+\frac{1}{\Delta t} \int_{\partial K} \widehat{q}^{n+1} \mathcal{D} \boldsymbol{w} \cdot \boldsymbol{v} d s, \\
& -\frac{1}{\Delta t} \int_{K} r^{n+1} \mathcal{D} \phi d K=-\frac{1}{\Delta t} \int_{K}\left(\left(\phi^{n+1}\right)^{3}+(1-\epsilon) \phi^{n+1}\right) \mathcal{D} \phi d K .
\end{aligned}
$$

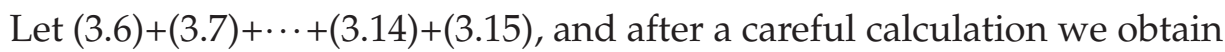

$$
\begin{array}{r}
\frac{1}{\Delta t} \int_{\Omega}\left(\frac{1}{M} \mathcal{D} s \cdot s^{n+1}+\mathcal{D}\right. \\
\left.q q^{n+1}-2 \mathcal{D} w \cdot w^{n}\right) d x+\int_{\Omega} \frac{\beta}{M} s^{n+1} \cdot s^{n+1} d x \\
+\frac{1}{\Delta t} \int_{\Omega}\left(\left(\phi^{n+1}\right)^{3}+(1-\epsilon) \phi^{n+1}\right) \mathcal{D} \phi d x=0 .
\end{array}
$$

with the help of the alternating numerical fluxes (3.3). Notice that

$$
\begin{aligned}
\left(\left(\phi^{n+1}\right)^{3}+(1-\epsilon) \phi^{n+1}\right) \mathcal{D} \phi= & \frac{1}{4}\left(\phi^{n+1}\right)^{4}+\frac{1-\epsilon}{2}\left(\phi^{n+1}\right)^{2}-\frac{1}{4}\left(\phi^{n}\right)^{4}-\frac{1-\epsilon}{2}\left(\phi^{n}\right)^{2} \\
& +\frac{1}{4}\left(2-2 \epsilon+2\left(\phi^{n+1}\right)^{2}+\left(\phi^{n}+\phi^{n+1}\right)^{2}\right)\left(\phi^{n+1}-\phi^{n}\right)^{2} .
\end{aligned}
$$


Therefore we have

$$
\begin{aligned}
& \frac{1}{\Delta t} \int_{\Omega}\left(\frac{1}{M} \mathcal{D} s \cdot s^{n+1}+\mathcal{D} q q^{n+1}-2 \mathcal{D} \boldsymbol{w} \cdot \boldsymbol{w}^{n}\right) d x \\
& \quad+\frac{1}{\Delta t} \int_{\Omega}\left(\frac{1}{4}\left(\phi^{n+1}\right)^{4}+\frac{1-\epsilon}{2}\left(\phi^{n+1}\right)^{2}-\frac{1}{4}\left(\phi^{n}\right)^{4}-\frac{1-\epsilon}{2}\left(\phi^{n}\right)^{2}\right) d x \leq 0
\end{aligned}
$$

which implies the energy stability result (3.5).

Lemma 3.1. [19] For any $u_{h} \in V_{h}^{0}:=\left\{v \in V_{h}:(v, 1)=0\right\}$, we have

$$
\left\|u_{h}\right\|_{\infty} \leq C\left(1+\left\|z_{h}\right\|\right) \sqrt{\log \left(1+\left\|\Delta_{h} u_{h}\right\|\right)},
$$

where $\Delta_{h}$ is the "discrete Laplacian" operator and $z_{h}$ satisfies

$$
\int_{K} z_{h} \cdot \boldsymbol{\theta} d K=-\int_{K} u_{h} \nabla \cdot \boldsymbol{\theta} d K+\int_{\partial K} \widehat{u_{h}} \boldsymbol{\theta} \cdot \boldsymbol{v} d s, \quad \boldsymbol{\theta} \in \Sigma_{h}^{d} .
$$

Theorem 3.1 (Estimate for $\left\|\phi^{n}\right\|_{\infty}$ ). The solution to the LDG scheme (3.2) satisfies

$$
\begin{aligned}
& \left\|\phi^{n}\right\| \leq C \\
& \left\|q^{n}\right\| \leq C \\
& \left\|\boldsymbol{w}^{n}\right\| \leq C \\
& \left\|\phi^{n}\right\|_{\infty} \leq C
\end{aligned}
$$

where $C>0$ is independent of $h$.

Proof. From the energy stability result (3.5), we have

$$
\mathcal{E}_{h}\left(q^{n}, \boldsymbol{w}^{n}, \boldsymbol{s}^{n}, \phi^{n}\right) \leq \mathcal{E}_{h}\left(q^{0}, \boldsymbol{w}^{0}, \boldsymbol{s}^{0}, \phi^{0}\right) \leq C,
$$

where the last inequality is because of the choice of the initial condition $q^{0}=P q(x, 0)$, $w^{0}=\Pi w(x, 0), s^{0}=\Pi s(x, 0), \phi^{0}=P \phi(x, 0)$, where $P$ and $\Pi$ are the standard $L^{2}$ projection.

$$
\begin{aligned}
\mathcal{E}_{h}\left(q^{n}, \boldsymbol{w}^{n}, \boldsymbol{s}^{n}, \phi^{n}\right) & =\int_{\Omega}\left(\frac{1}{2}\left(q^{n}\right)^{2}-\boldsymbol{w}^{n} \cdot \boldsymbol{w}^{n}+\frac{1}{2 M} \boldsymbol{s}^{n} \cdot \boldsymbol{s}^{n}+\frac{1}{4}\left(\phi^{n}\right)^{4}+\frac{1-\epsilon}{2}\left(\phi^{n}\right)^{2}\right) d x \\
& \geq \int_{\Omega}\left(\frac{2-\epsilon}{2}\left(\phi^{n}\right)^{2}+\frac{1}{2}\left(q^{n}\right)^{2}+\frac{1}{2 M} \boldsymbol{s}^{n} \cdot \boldsymbol{s}^{n}-\boldsymbol{w}^{n} \cdot \boldsymbol{w}^{n}\right) d \boldsymbol{x} .
\end{aligned}
$$

For Eqs. (3.2g) and (3.2h) of the LDG scheme, choosing the test functions $\varphi_{4}=\phi^{n}, \boldsymbol{\theta}_{4}=\boldsymbol{w}^{n}$ at time level $t^{n}$ leads to

$$
\begin{aligned}
\int_{K} q^{n} \phi^{n} d K & =-\int_{K} \boldsymbol{w}^{n} \cdot \nabla \phi^{n} d K+\int_{\partial K} \widehat{\boldsymbol{w}}^{n} \cdot \boldsymbol{v} \phi^{n} d s, \\
\int_{K} \boldsymbol{w}^{n} \cdot \boldsymbol{w}^{n} d K & =-\int_{K} \phi^{n} \nabla \cdot \boldsymbol{w}^{n} d K+\int_{\partial K} \widehat{\phi}^{n} \boldsymbol{w}^{n} \cdot \boldsymbol{v} d s .
\end{aligned}
$$


Let (3.17)+(3.18), we have

$$
\int_{\Omega} w^{n} \cdot w^{n} d x=-\int_{\Omega} q^{n} \phi^{n} d x \leq \int_{\Omega}\left(\frac{\delta}{2}\left(q^{n}\right)^{2}+\frac{1}{2 \delta}\left(\phi^{n}\right)^{2}\right) d x .
$$

Thus

$$
\begin{aligned}
\mathcal{E}_{h}\left(q^{n}, \boldsymbol{w}^{n}, \boldsymbol{s}^{n}, \phi^{n}\right) & \geq \int_{\Omega}\left(\left(\frac{2-\epsilon}{2}-\frac{1}{2 \delta}\right)\left(\phi^{n}\right)^{2}+\left(\frac{1-\delta}{2}\right)\left(q^{n}\right)^{2}+\frac{1}{2 M} s^{n} \cdot \boldsymbol{s}^{n}\right) d x \\
& \geq \int_{\Omega}\left(C\left(\left(\phi^{n}\right)^{2}+\left(q^{n}\right)^{2}\right)+\frac{1}{2 M} s^{n} \cdot s^{n}\right) d x \\
& \geq \int_{\Omega}\left(C\left(\frac{\left(\phi^{n}\right)^{2}+\left(q^{n}\right)^{2}}{2}+\boldsymbol{w}^{n} \cdot \boldsymbol{w}^{n}\right)+\frac{1}{2 M} \boldsymbol{s}^{n} \cdot \boldsymbol{s}^{n}\right) d x,
\end{aligned}
$$

which implies

$$
\left\|\phi^{n}\right\| \leq C, \quad\left\|q^{n}\right\| \leq C, \quad\left\|\boldsymbol{w}^{n}\right\| \leq C .
$$

Using Lemma 3.1, we have

$$
\left\|\phi^{n}\right\|_{\infty} \leq C\left(1+\left\|w^{n}\right\|\right) \sqrt{\log \left(1+\left\|\Delta_{h} \phi^{n}\right\|\right)} .
$$

By the definition of the discrete Laplacian operator, we have

$$
\int_{K} q^{n} \zeta d K=\int_{K} \Delta_{h} \phi^{n} \zeta d K, \quad \zeta \in V_{h} .
$$

Choosing $\zeta=\Delta_{h} \phi^{n}$ in (3.21), we get

$$
\left\|\Delta_{h} \phi^{n}\right\|^{2}=\int_{\Omega} q^{n} \Delta_{h} \phi^{n} d x \leq\left\|q^{n}\right\| \cdot\left\|\Delta_{h} \phi^{n}\right\| .
$$

Combining (3.20) and (3.22), we can obtain the result $\left\|\phi^{n}\right\|_{\infty} \leq C$.

\subsection{Second order fully discrete LDG scheme}

To construct the LDG method for the second order convex splitting scheme (2.9), we first rewrite it into the system form:

$$
\begin{aligned}
\frac{\phi^{n+1}-\phi^{n}}{\Delta t} & =\nabla \cdot s^{n+\frac{1}{2},} \\
s^{n+1} & =\nabla u^{n+1}, \\
\frac{\nabla \cdot s^{n+1}-\nabla \cdot s^{n}}{\Delta t} & =M \nabla \cdot p^{n+1}-\beta \nabla \cdot s^{n+\frac{1}{2},} \\
p^{n+1} & =\nabla\left(r^{n+1}+3 q^{n}-q^{n-1}+q_{2}^{n+\frac{1}{2}}\right),
\end{aligned}
$$




$$
\begin{aligned}
q_{2}^{n+1} & =\nabla \cdot \boldsymbol{q}_{1}^{n+1}, \\
\boldsymbol{q}_{1}^{n+1} & =\nabla q^{n+1}, \\
q^{n+1} & =\nabla \cdot \boldsymbol{w}^{n+1}, \\
\boldsymbol{w}^{n+1} & =\nabla \phi^{n+1}, \\
r^{n+1} & =(1-\epsilon) \phi^{n+\frac{1}{2}}+\frac{1}{2}\left(\left(\phi^{n+1}\right)^{2}+\left(\phi^{n}\right)^{2}\right) \phi^{n+\frac{1}{2}},
\end{aligned}
$$

which contains only first order spatial derivatives, and

$$
s^{n+\frac{1}{2}}=\frac{1}{2}\left(s^{n+1}+s^{n}\right), \quad q_{2}^{n+\frac{1}{2}}=\frac{1}{2}\left(q_{2}^{n+1}+q_{2}^{n}\right) .
$$

The LDG scheme for the system (3.23) is defined as: Find $\phi^{n+1}, u^{n+1}, q_{2}^{n+1}, q^{n+1}, r^{n+1} \in$ $V_{h}$ and $\boldsymbol{p}^{n+1}, \boldsymbol{s}^{n+1}, \boldsymbol{q}_{1}^{n+1}, \boldsymbol{w}^{n+1} \in \Sigma_{h^{\prime}}^{d}$, such that, for all test functions $\varphi_{1}, \varphi_{2}, \varphi_{3}, \varphi_{4}, \varphi_{5} \in V_{h}$ and $\boldsymbol{\theta}_{1}, \boldsymbol{\theta}_{2}, \boldsymbol{\theta}_{3}, \boldsymbol{\theta}_{4} \in \Sigma_{h}^{d}$, we have

$$
\begin{aligned}
\frac{1}{\Delta t} \int_{K} \mathcal{D} \phi \varphi_{1} d K= & -\int_{K} s^{n+\frac{1}{2}} \cdot \nabla \varphi_{1} d K+\int_{\partial K} \widehat{\boldsymbol{s}}^{n+\frac{1}{2}} \cdot \boldsymbol{v} \varphi_{1} d s \\
\int_{K} s^{n+1} \cdot \boldsymbol{\theta}_{1} d K= & -\int_{K} u^{n+1} \nabla \cdot \boldsymbol{\theta}_{1} d K+\int_{\partial K} \widehat{u}^{n+1} \boldsymbol{\theta}_{1} \cdot \boldsymbol{v} d s \\
0= & \int_{K} \frac{\boldsymbol{s}^{n+1}-\boldsymbol{s}^{n}}{\Delta t} \cdot \nabla \varphi_{2} d K-\int_{\partial K} \frac{\left(\widehat{\boldsymbol{s}}^{n+1}-\widehat{\boldsymbol{s}}^{n}\right) \cdot \boldsymbol{v}}{\Delta t} \varphi_{2} d s \\
& -\int_{K}\left(M \boldsymbol{p}^{n+1}-\beta \boldsymbol{s}^{n+\frac{1}{2}}\right) \cdot \nabla \varphi_{2} d K+\int_{\partial K}\left(M \widehat{\boldsymbol{p}}^{n+1}-\beta \widehat{\boldsymbol{s}}^{n+\frac{1}{2}}\right) \cdot \boldsymbol{v} \varphi_{2} d s \\
\int_{K} \boldsymbol{p}^{n+1} \cdot \boldsymbol{\theta}_{2} d K= & -\int_{K}\left(r^{n+1}+3 q^{n}-q^{n-1}+q_{2}^{n+\frac{1}{2}}\right) \nabla \cdot \boldsymbol{\theta}_{2} d K \\
& +\int_{\partial K}\left(\widehat{r}^{n+1}+3 \widehat{q}^{n}-\widehat{q}^{n-1}+\widehat{q}_{2}^{n+\frac{1}{2}}\right) \boldsymbol{\theta}_{2} \cdot \boldsymbol{v} d s \\
\int_{K} q_{2}^{n+1} \varphi_{3} d K= & -\int_{K} \boldsymbol{q}_{1}^{n+1} \cdot \nabla \varphi_{3} d K+\int_{\partial K} \widehat{\boldsymbol{q}}_{1}^{n+1} \cdot \boldsymbol{v} \varphi_{3} d S= \\
\int_{K} q^{n+1} \varphi_{4} d K= & -\int_{K} q^{n+1} \nabla \cdot \boldsymbol{\theta}_{3} d K+\int_{\partial K} \widehat{q}^{n+1} \cdot \nabla \varphi_{4} d K+\int_{\partial K} \widehat{\boldsymbol{w}}^{n+1} \cdot \boldsymbol{v} \varphi_{4} d s \\
\int_{K} \boldsymbol{w}^{n+1} \cdot \boldsymbol{\theta}_{4} d K= & -\int_{K} \phi^{n+1} \nabla \cdot \boldsymbol{\theta}_{4} d K+\int_{\partial K} \widehat{\phi}^{n+1} \boldsymbol{\theta}_{4} \cdot \boldsymbol{v} d s \\
\int_{K} r^{n+1} \varphi_{5} d K= & \int_{K}\left((1-\epsilon) \phi^{n+\frac{1}{2}}+\frac{1}{2}\left(\left(\phi^{n+1}\right)^{2}+\left(\phi^{n}\right)^{2}\right) \phi^{n+\frac{1}{2}}\right) \varphi_{5} d K
\end{aligned}
$$

The second order fully discrete LDG scheme (3.24) is also mass conservative. Next, we will prove the unconditional energy stability for the second order fully discrete LDG scheme (3.24). 
Proposition 3.3. (Energy stability for the second order fully discrete LDG scheme). The solution to the LDG scheme (3.24) with the numerical fluxes (3.3) and the periodic boundary condition satisfies the energy stability

$$
\mathcal{F}_{h}\left(q^{n+1}, \boldsymbol{w}^{n+1}, \boldsymbol{w}^{n}, \boldsymbol{s}^{n+1}, \phi^{n+1}\right)-\mathcal{F}_{h}\left(q^{n}, \boldsymbol{w}^{n}, \boldsymbol{w}^{n-1}, \boldsymbol{s}^{n}, \phi^{n}\right) \leq 0,
$$

where

$$
\begin{aligned}
\mathcal{F}_{h}\left(q, w_{1}, w_{2}, s, \phi\right)= & \int_{\Omega}\left(\frac{1}{2}\left(q^{2}+\frac{1}{M} s \cdot s+\left(w_{1}-w_{2}\right) \cdot\left(w_{1}-w_{2}\right)\right)\right. \\
& \left.-w_{1} \cdot w_{1}+\frac{1}{4} \phi^{4}+\frac{1-\epsilon}{2} \phi^{2}\right) d x
\end{aligned}
$$

namely

$$
\begin{aligned}
\mathcal{F}_{h}\left(q^{n+1}, \boldsymbol{w}^{n+1}, \boldsymbol{w}^{n}, \mathbf{s}^{n+1}, \phi^{n+1}\right)= & \mathcal{E}_{h}\left(q^{n+1}, \boldsymbol{w}^{n+1}, \mathbf{s}^{n+1}, \phi^{n+1}\right) \\
& +\frac{1}{2} \int_{\Omega}\left(\boldsymbol{w}^{n+1}-\boldsymbol{w}^{n}\right) \cdot\left(\boldsymbol{w}^{n+1}-\boldsymbol{w}^{n}\right) d x .
\end{aligned}
$$

Proof. Let $\boldsymbol{q}_{1}^{n+\frac{1}{2}}:=\frac{1}{2}\left(\boldsymbol{q}_{1}^{n+1}+\boldsymbol{q}_{1}^{n}\right)$ and $q^{n+\frac{1}{2}}:=\frac{1}{2}\left(q^{n+1}+q^{n}\right)$. For (3.24b), (3.24e), (3.24f), taking the sum between time level $t^{n+1}$ and $t^{n}$, and choosing test functions

$$
\boldsymbol{\theta}_{1}=\frac{1}{M \Delta t} \mathcal{D} \boldsymbol{s}+\frac{\beta}{M} s^{n+\frac{1}{2}}-p^{n+1}, \varphi_{3}=-\frac{\mathcal{D} \phi}{\Delta t}, \boldsymbol{\theta}_{3}=\frac{\mathcal{D} \boldsymbol{w}}{\Delta t},
$$

respectively, we have

$$
\begin{aligned}
& \int_{K} s^{n+\frac{1}{2}} \cdot\left(\frac{1}{M \Delta t} \mathcal{D} s+\frac{\beta}{M} s^{n+\frac{1}{2}}-p^{n+1}\right) d K=-\int_{K} u^{n+\frac{1}{2}} \nabla \cdot\left(\frac{1}{M \Delta t} \mathcal{D} s+\frac{\beta}{M} s^{n+\frac{1}{2}}-p^{n+1}\right) d K \\
&+\int_{\partial K} \widehat{u}^{n+\frac{1}{2}}\left(\frac{1}{M \Delta t} \mathcal{D} s+\frac{\beta}{M} s^{n+\frac{1}{2}}-p^{n+1}\right) \cdot v d s \\
&-\frac{1}{\Delta t} \int_{K} q_{2}^{n+\frac{1}{2}} \mathcal{D} \phi d K=\frac{1}{\Delta t} \int_{K} q_{1}^{n+\frac{1}{2}} \cdot \nabla \mathcal{D} \phi d K-\frac{1}{\Delta t} \int_{\partial K} \widehat{\boldsymbol{q}}_{1}^{n+\frac{1}{2}} \cdot v \mathcal{D} \phi d s \\
& \frac{1}{\Delta t} \int_{K} q_{1}^{n+\frac{1}{2}} \cdot \mathcal{D} w d K=-\frac{1}{\Delta t} \int_{K} q^{n+\frac{1}{2}} \nabla \cdot \mathcal{D} w d K+\frac{1}{\Delta t} \int_{\partial K} \widehat{q}^{n+\frac{1}{2}} \mathcal{D} \boldsymbol{w} \cdot \boldsymbol{v} d s
\end{aligned}
$$

For $(3.24 \mathrm{~g}),(3.24 \mathrm{~h})$, taking the difference between time level $t^{n+1}$ and $t^{n}$, and choosing test functions

respectively, we have

$$
\varphi_{4}=\frac{1}{\Delta t} q^{n+\frac{1}{2}}, \quad \theta_{4}=\frac{1}{\Delta t}\left(\boldsymbol{w}^{n-1}-3 \boldsymbol{w}^{n}-q_{1}^{n+\frac{1}{2}}\right)
$$

$$
\begin{aligned}
\frac{1}{\Delta t} \int_{K} \mathcal{D} q q^{n+\frac{1}{2}} d K=-\frac{1}{\Delta t} \int_{K} \mathcal{D} \boldsymbol{w} \cdot \nabla q^{n+\frac{1}{2}} & d K+\frac{1}{\Delta t} \int_{\partial K} \mathcal{D} \widehat{\boldsymbol{w}} \cdot \boldsymbol{v} q^{n+\frac{1}{2}} d s \\
\frac{1}{\Delta t} \int_{K} \mathcal{D} \boldsymbol{w} \cdot\left(\boldsymbol{w}^{n-1}-3 \boldsymbol{w}^{n}-\boldsymbol{q}_{1}^{n+\frac{1}{2}}\right) d K= & -\frac{1}{\Delta t} \int_{K} \mathcal{D} \phi \nabla \cdot\left(\boldsymbol{w}^{n-1}-3 \boldsymbol{w}^{n}-\boldsymbol{q}_{1}^{n+\frac{1}{2}}\right) d K \\
& +\frac{1}{\Delta t} \int_{\partial K} \mathcal{D} \widehat{\phi}\left(\boldsymbol{w}^{n-1}-3 \boldsymbol{w}^{n}-\boldsymbol{q}_{1}^{n+\frac{1}{2}}\right) \cdot \boldsymbol{v} d s .
\end{aligned}
$$


For $(3.24 \mathrm{~g})$, choosing the test function $\varphi_{4}=-\frac{3 \mathcal{D} \phi}{\Delta t}$ at time level $t^{n}$ and $\varphi_{4}=\frac{\mathcal{D} \phi}{\Delta t}$ at time level $t^{n-1}$ show that

$$
\begin{aligned}
& -\frac{3}{\Delta t} \int_{K} q^{n} \mathcal{D} \phi d K=\frac{3}{\Delta t} \int_{K} w^{n} \cdot \nabla \mathcal{D} \phi d K-\frac{3}{\Delta t} \int_{\partial K} \widehat{\boldsymbol{w}}^{n} \cdot \boldsymbol{v} \mathcal{D} \phi d s, \\
& \frac{1}{\Delta t} \int_{K} q^{n-1} \mathcal{D} \phi d K=-\frac{1}{\Delta t} \int_{K} \boldsymbol{w}^{n-1} \cdot \nabla \mathcal{D} \phi d K+\frac{1}{\Delta t} \int_{\partial K} \widehat{\boldsymbol{w}}^{n-1} \cdot \boldsymbol{v} \mathcal{D} \phi d s .
\end{aligned}
$$

For (3.24a), (3.24c), (3.24d) and (3.24i), taking the test functions

$$
\varphi_{1}=r^{n+1}+3 q^{n}-q^{n-1}+q_{2}^{n+\frac{1}{2}}, \varphi_{2}=-\frac{1}{M} u^{n+\frac{1}{2}}, \theta_{2}=s^{n+\frac{1}{2}}, \varphi_{5}=-\frac{\mathcal{D} \phi}{\Delta t},
$$

respectively, we have

$$
\begin{aligned}
& \frac{1}{\Delta t} \int_{K} \mathcal{D} \phi\left(r^{n+1}+3 q^{n}-q^{n-1}+q_{2}^{n+\frac{1}{2}}\right) d K=-\int_{K}\left(s^{n+\frac{1}{2}} \cdot \nabla\left(r^{n+1}+3 q^{n}-q^{n-1}+q_{2}^{n+\frac{1}{2}}\right)\right) d K \\
& \quad+\int_{\partial K} \widehat{\boldsymbol{s}}^{n+\frac{1}{2}} \cdot \boldsymbol{v}\left(r^{n+1}+3 q^{n}-q^{n-1}+q_{2}^{n+\frac{1}{2}}\right) d s \\
0 & -\int_{K} \frac{\mathcal{D} \boldsymbol{s}}{M \Delta t} \cdot \nabla u^{n+\frac{1}{2}} d K+\int_{\partial K} \frac{\mathcal{D} \widehat{\boldsymbol{s}} \cdot \boldsymbol{v}}{M \Delta t} u^{n+\frac{1}{2}} d s \\
& +\int_{K}\left(\boldsymbol{p}^{n+1}-\frac{\beta}{M} \boldsymbol{s}^{n+\frac{1}{2}}\right) \cdot \nabla u^{n+\frac{1}{2}} d K-\int_{\partial K}\left(\widehat{\boldsymbol{p}}^{n+1}-\frac{\beta}{M} \widehat{\boldsymbol{s}}^{n+\frac{1}{2}}\right) \cdot v u^{n+\frac{1}{2}} d s \\
& \int_{K} \boldsymbol{p}^{n+1} \cdot \boldsymbol{s}^{n+\frac{1}{2}} d K=-\int_{K}\left(r^{n+1}+3 q^{n}-q^{n-1}+q_{2}^{n+\frac{1}{2}}\right) \nabla \cdot \boldsymbol{s}^{n+\frac{1}{2}} d K \\
& +\int_{\partial K}\left(\widehat{r}^{n+1}+3 \widehat{q}^{n}-\widehat{q}^{n-1}+\widehat{q}_{2}^{n+\frac{1}{2}}\right) \boldsymbol{s}^{n+\frac{1}{2}} \cdot v d s, \\
& -\frac{1}{\Delta t} \int_{K} r^{n+1} \mathcal{D} \phi d K=-\frac{1}{\Delta t} \int_{K}\left((1-\epsilon) \phi^{n+\frac{1}{2}}+\frac{1}{2}\left(\left(\phi^{n+1}\right)^{2}+\left(\phi^{n}\right)^{2}\right) \phi^{n+\frac{1}{2}}\right) \mathcal{D} \phi d K
\end{aligned}
$$

Let (3.27) $+(3.28)+\cdots+(3.36)+(3.37)$, with the help of the alternating numerical fluxes (3.3), and after a careful calculation, we obtain

$$
\begin{aligned}
& \frac{1}{2 \Delta t} \int_{\Omega}\left(\left(q^{n+1}\right)^{2}-\left(q^{n}\right)^{2}+\frac{1}{M} s^{n+1} \cdot s^{n+1}-\frac{1}{M} s^{n} \cdot s^{n}\right) d x+\frac{\beta}{4 M} \int_{\Omega}\left(s^{n+1}+s^{n}\right) \cdot\left(s^{n+1}+s^{n}\right) d x \\
& \quad+\frac{1}{\Delta t} \int_{\Omega}\left(\frac{1-\epsilon}{2}\left(\left(\phi^{n+1}\right)^{2}-\left(\phi^{n}\right)^{2}\right)+\frac{1}{4}\left(\left(\phi^{n+1}\right)^{4}-\left(\phi^{n}\right)^{4}\right)\right) d x \\
& \quad+\frac{1}{\Delta t} \int_{\Omega}\left(w^{n-1}-3 w^{n}\right) \cdot\left(w^{n+1}-w^{n}\right) d x=0 .
\end{aligned}
$$

Notice that

$$
\begin{aligned}
& \left(\boldsymbol{w}^{n-1}-3 \boldsymbol{w}^{n}\right) \cdot\left(\boldsymbol{w}^{n+1}-\boldsymbol{w}^{n}\right) \\
= & -\boldsymbol{w}^{n+1} \cdot \boldsymbol{w}^{n+1}+\boldsymbol{w}^{n} \cdot \boldsymbol{w}^{n}+\frac{1}{2}\left(\boldsymbol{w}^{n+1}-2 \boldsymbol{w}^{n}+\boldsymbol{w}^{n-1}\right) \cdot\left(\boldsymbol{w}^{n+1}-2 \boldsymbol{w}^{n}+\boldsymbol{w}^{n-1}\right) \\
& +\frac{1}{2}\left(\boldsymbol{w}^{n+1}-\boldsymbol{w}^{n}\right) \cdot\left(\boldsymbol{w}^{n+1}-\boldsymbol{w}^{n}\right)-\frac{1}{2}\left(\boldsymbol{w}^{n}-\boldsymbol{w}^{n-1}\right) \cdot\left(\boldsymbol{w}^{n}-\boldsymbol{w}^{n-1}\right) .
\end{aligned}
$$


Therefore, we have

$$
\mathcal{F}_{h}\left(q^{n+1}, \boldsymbol{w}^{n+1}, \boldsymbol{w}^{n}, \boldsymbol{s}^{n+1}, \phi^{n+1}\right)-\mathcal{F}_{h}\left(q^{n}, \boldsymbol{w}^{n}, \boldsymbol{w}^{n-1}, \boldsymbol{s}^{n}, \phi^{n}\right) \leq 0 .
$$

This completes the proof.

Remark 3.1. The unconditional energy stability of the convex splitting schemes are proved. But as for the unique solvability and the convergence analysis, the proofs are much more elusive in the LDG framework. Even though the auxiliary variables in the LDG method give the easy treatment for nonlinear and high order derivatives terms, the theoretical analysis of solvability for the LDG method is more troublesome because of the auxiliary variables. In addition, the main technical difficulty of convergence analysis is the lack of control on the auxiliary variables in the LDG method which are approximations to the derivatives of the solution, and the lack of control on the interface boundary terms. We will leave them as our future work.

\subsection{First order linear fully discrete LDG scheme}

The LDG method to solve the first order linear scheme (2.12) is similar as (3.2), except replacing (3.2i) by

$$
\int_{K} r^{n+1} \varphi_{5} d K=\int_{K}\left(U^{n+1} \phi^{n}+(1-\epsilon) \phi^{n+1}\right) \varphi_{5} d K
$$

and adding

$$
\int_{K} \frac{U^{n+1}-U^{n}}{\Delta t} \zeta d K=\int_{K} 2 \phi^{n} \frac{\phi^{n+1}-\phi^{n}}{\Delta t} \zeta d K, \quad \zeta \in V_{h} .
$$

In the following, we will prove the energy stability for the fully-discrete LDG scheme to solve (2.12) with the choice of the numerical flux (3.3).

Proposition 3.4. (Energy stability for the first order linear LDG scheme). The solution to the first order linear LDG scheme with the numerical fluxes (3.3) and the periodic boundary condition satisfies the energy stability

$$
\mathcal{E}_{h}\left(q^{n+1}, \boldsymbol{w}^{n+1}, \boldsymbol{s}^{n+1}, \phi^{n+1}, U^{n+1}\right)-\mathcal{E}_{h}\left(q^{n}, \boldsymbol{w}^{n}, \mathbf{s}^{n}, \phi^{n}, U^{n}\right) \leq 0,
$$

where

$$
\mathcal{E}_{h}(q, w, s, \phi, U)=\int_{\Omega}\left(\frac{1}{2} q^{2}-w \cdot w+\frac{1}{2 M} s \cdot s+\frac{1}{4} U^{2}+\frac{1-\epsilon}{2} \phi^{2}\right) d x
$$

Proof. For (3.38), choosing the test function $\varphi_{5}=-\frac{1}{\Delta t} \mathcal{D} \phi$ leads to

$$
-\frac{1}{\Delta t} \int_{K} r^{n+1} \mathcal{D} \phi d K=-\frac{1}{\Delta t} \int_{K}\left(\phi^{n} U^{n+1}+(1-\epsilon) \phi^{n+1}\right) \mathcal{D} \phi d K .
$$


Taking the test function $\zeta=\frac{1}{2} U^{n+1}$ in (3.39) and using the identity

$$
2(a-b, a)=|a|^{2}-|b|^{2}+|a-b|^{2},
$$

we can get

$$
\frac{1}{4 \Delta t} \int_{K}\left(\left(U^{n+1}\right)^{2}-\left(U^{n}\right)^{2}+\left(U^{n+1}-U^{n}\right)^{2}\right) d K=\frac{1}{\Delta t} \int_{K} \phi^{n} U^{n+1} \mathcal{D} \phi d K .
$$

Let (3.6)+(3.7) $+\ldots+(3.14)+(3.41)+(3.42)$ and use the same technique as the proof of Proposition (3.2), we can get the energy stability result (3.40) immediately.

Remark 3.2. For the second order convex splitting scheme (2.9) and the first order linear scheme (2.12), we can also obtain the estimation of $\left\|\phi^{n}\right\|_{\infty}$ similar as Theorem 3.1 based on the corresponding energy stability result. We will omit the proof in this paper.

Remark 3.3. Eqs. (3.2), (3.24) and (2.12) at the implicit time level are nonlinear or linear, we will employ a nonlinear full approximation storage (FAS) multigrid method [4] or linear multigrid solver to solve the equations. The theoretical analysis of the convergence of multigrid solvers is more troublesome. However, numerical experiments are presented to show that the multigrid solver is robust and converges with optimal (or near optimal) complexity.

\section{A high order and adaptive time-stepping strategy}

The most important property of LDG methods is high order accurate, which motivates us to develop high order temporal accuracy scheme to get the goal of obtaining high order accuracy in both space and time together with robust stability conditions.

The proposed first order and second order schemes are unconditionally energy stable, thus allowing for large time stepping. However, the schemes are only first order or second order accurate in time. In order to improve the temporal accuracy, the semiimplicit SDC method combining with the first order convex splitting scheme (3.2) will be employed to solve the MPFC equation.

\subsection{Semi-implicit spectral deferred correction method}

The SDC method is driven iteratively by the chosen low order method, such as the first order convex splitting scheme (3.2) here. An advantage of the SDC method is that it is a one step method and can be constructed easily and systematically for any order of accuracy. For convenience, the convex splitting scheme (3.2) can be written as

$$
\left\{\begin{array}{l}
\phi^{n+1}=\phi^{n}+\Delta t F\left(u^{n+1}\right), \\
\Delta u^{n+1}=\Delta u^{n}+\Delta t\left(L_{1}\left(u^{n+1}\right)+L_{2}\left(\phi^{n+1}\right)+N\left(\phi^{n}\right)\right),
\end{array}\right.
$$


where $F(u), L_{1}(u)$ represent the linearly implicit part, $L_{2}(\phi)$ represents the nonlinearly implicit part and $N(\phi)$ represents the linearly explicit part of the scheme, namely

$$
\begin{aligned}
& F(u)=\Delta u, \quad L_{1}(u)=-\beta \Delta u, \\
& L_{2}(\phi)=M \Delta\left(\phi^{3}+(1-\epsilon) \phi+\Delta^{2} \phi\right), \quad N(\phi)=2 M \Delta(\Delta \phi) .
\end{aligned}
$$

Suppose now the time interval $[0, T]$ is divided into $M$ intervals by the partition $0=t_{0}<$ $t_{1}<\ldots<t_{n}<\ldots<t_{M}=T$. Let $\Delta t_{n}=t_{n+1}-t_{n}$ and $u^{n}$ denotes the numerical approximation of $u\left(t_{n}\right)$, with $u_{0}=u(0)$. Then divide the time interval $\left[t_{n}, t_{n+1}\right]$ into $P$ subintervals by choosing the points $t_{n, m}$ for $m=0,1, \cdots, P$ such that $t_{n}=t_{n, 0}<t_{n, 1}<\cdots<t_{n, m}<\cdots<t_{n, P}=t_{n+1}$. Let $\Delta t_{n, m}=t_{n, m+1}-t_{n, m}$ and $u_{n, m}^{k}$ denotes the $k^{t h}$ order approximation to $u\left(t_{n, m}\right)$. The points $\left\{t_{n, m}\right\}_{m=0}^{P}$ can be chosen to be the Tchebychev Gauss-Lobatto nodes on $\left[t_{n}, t_{n+1}\right]$ to avoid the instability of approximation at equispaced nodes for high order accuracy. We can also choose the Gauss nodes, or Legendre Gauss-Radau nodes or Legendre GaussLobatto nodes. Starting from $u^{n}$ and $\phi^{n}$, we give the algorithm to calculate $u^{n+1}$ and $\phi^{n+1}$ in the following.

\section{Compute the initial approximation:}

$u_{n, 0}^{1}=u^{n}$ and $\phi_{n, 0}^{1}=\phi^{n}$.

Use the convex splitting scheme (4.1) to compute a first order accurate approximate solution $u^{1}$ and $\phi^{1}$ at the nodes $\left\{t_{n, m}\right\}_{m=1}^{P}$, i.e.

For $m=0, \cdots, P-1$,

$$
\left\{\begin{array}{l}
\phi_{n, m+1}^{1}=\phi_{n, m}^{1}+\Delta t_{n, m} F\left(u_{n, m+1}^{1}\right), \\
\Delta u_{n, m+1}^{1}=\Delta u_{n, m}^{1}+\Delta t_{n, m}\left(L_{1}\left(u_{n, m+1}^{1}\right)+L_{2}\left(\phi_{n, m+1}^{1}\right)+N\left(\phi_{n, m}^{1}\right)\right) .
\end{array}\right.
$$

\section{Compute successive corrections:}

For $k=1, \cdots, K$,

$u_{n, 0}^{k+1}=u^{n}$ and $\phi_{n, 0}^{k+1}=\phi^{n}$.

For $m=0, \cdots, P-1$,

$$
\left\{\begin{aligned}
\phi_{n, m}^{k+1}= & \phi_{n, m}^{k+1}+\Delta t_{n, m}\left(F\left(u_{n, m+1}^{k+1}\right)-F\left(u_{n, m+1}^{k}\right)\right)+I_{m}^{m+1}\left(F\left(u^{k}\right)\right), \\
\Delta u_{n, m+1}^{k+1}= & \Delta u_{n, m}^{k+1}+\Delta t_{n, m}\left(L_{1}\left(u_{n, m+1}^{k+1}\right)-L_{1}\left(u_{n, m+1}^{k}\right)+L_{2}\left(\phi_{n, m+1}^{k+1}\right)-L_{2}\left(\phi_{n, m+1}^{k}\right)\right) \\
& +\Delta t_{n, m}\left(N\left(\phi_{n, m}^{k+1}\right)-N\left(\phi_{n, m}^{k}\right)\right)+I_{m}^{m+1}\left(L_{1}\left(u^{k}\right)+L_{2}\left(\phi^{k}\right)+N\left(\phi^{k}\right)\right),
\end{aligned}\right.
$$

where $I_{m}^{m+1}\left(F\left(u^{k}\right)\right)$ is the integral of the $P$-th degree interpolating polynomial on the $P+1$ points $\left(t_{n, m}, F\left(u_{n, m}^{k}\right)\right)_{m=0}^{P}$ over the subinterval $\left[t_{n, m}, t_{n, m+1}\right]$, which is the numerical quadrature approximation of

$$
\int_{t_{n, m}}^{t_{n, m+1}} F(\tau, u(\tau)) d \tau
$$

Finally we have $u^{n+1}=u_{n, P}^{K+1}$ and $\phi^{n+1}=\phi_{n, P}^{k+1}$. 
Remark 4.1. When $M(\phi)$ is degenerate, namely, the stiff and non-stiff components of the MPFC equation can not be well separated, the classical SDC method is not straightforward anymore. In this case, the novel semi-implicit SDC method proposed in our recent work [16] can be employed to achieve high order temporal accuracy. Actually, the SDC method described above is a special case of the method developed in [16].

\subsection{Adaptive time-stepping strategy}

In the previous subsection, we have proved that the semi-implicit schemes are unconditionally energy stable, which allows us using large time steps during the numerical simulations. However, constant large time steps may cause accuracy loss, except in the time intervals where the solution variation is relatively small. As we know, the energy is an important quantity in the MPFC equation and here the adaptive time steps are determined based on the time derivative of the energy similar to [27], which takes the following form

$$
\Delta t=\max \left(\Delta t_{\min }, \frac{\Delta t_{\max }}{\sqrt{1+\alpha\left|\mathcal{E}^{\prime}(t)\right|^{2}}}\right),
$$

where $\mathcal{E}$ is the energy functional defined in (2.6). The constant $\alpha$, which is used to adjust the level of adaptivity, is chosen in experience.

\section{$5 \quad$ Numerical experiments}

In this section, we present some numerical experiments for solving the MPFC equation. Specially, we employ the LDG spatial discretization coupled with the semi-implicit SDC method with adaptive time-stepping technique described in the previous sections. Each time step we solve the resulting algebraic equations by multigrid solver [4]. We first present the accuracy tests, which shows the expected optimal convergence rate and the efficiency of the proposed adaptive time-stepping technique. An energy stability test is then presented to show the pseudo energy (2.6) is indeed non-increasing. Finally, we give two long time simulations to illustrate the efficiency and capability of the proposed approaches.

Example 5.1. (Accuracy test and efficiency test of adaptive time-stepping strategy). Consider the MPFC equation (1.1) in the two-dimensional domain $\Omega=[0,2 \pi] \times[0,2 \pi]$. To verify the convergence rate, we add a source term to the MPFC equation such that the exact solution is

$$
\phi(x, y)=e^{-2 t} \sin (x) \sin (y) .
$$

Choose the parameters $\epsilon=0.025, M=1$ and $\beta=0.9$.

The $L^{2}$ and $L^{\infty}$ errors and the numerical orders of accuracy at time $T=0.5$ for the first order linear LDG scheme implemented with $\mathcal{P}^{0}$ elements is presented in Table 1 . It shows that the linear scheme is first order accurate in time. 
Table 1: Accuracy test for the MPFC equation at time $T=0.5$ obtained with the first order linear scheme. $\Delta t=0.1 \Delta x$.

\begin{tabular}{||c|c|cc|cc||}
\hline & $N$ & $L^{2}$ error & order & $L^{\infty}$ error & order \\
\hline \multirow{3}{*}{$\mathcal{P}^{0}$} & 16 & $3.62 \mathrm{E}-01$ & - & $1.30 \mathrm{E}-01$ & - \\
& 32 & $1.75 \mathrm{E}-01$ & 1.04 & $6.47 \mathrm{E}-02$ & 1.01 \\
& 64 & $8.59 \mathrm{E}-02$ & 1.03 & $3.16 \mathrm{E}-02$ & 1.03 \\
\hline
\end{tabular}

Table 2: Accuracy test for the MPFC equation at time $T=0.5$ obtained with the second order convex splitting method. $\Delta t=0.1 \Delta x$.

\begin{tabular}{||c|c|cc|cc||}
\hline & $N$ & $L^{2}$ error & order & $L^{\infty}$ error & order \\
\hline \multirow{3}{*}{$\mathcal{P}^{1}$} & 16 & $2.80 \mathrm{E}-02$ & - & $2.07 \mathrm{E}-02$ & - \\
& 32 & $6.93 \mathrm{E}-03$ & 2.01 & $5.24 \mathrm{E}-03$ & 1.98 \\
& 64 & $1.72 \mathrm{E}-03$ & 2.00 & $1.31 \mathrm{E}-03$ & 2.00 \\
\hline
\end{tabular}

Table 3: Accuracy test for the MPFC equation at time $T=0.5$ obtained with the third order semi-implicit SDC method. $\Delta t=0.1 \Delta x$.

\begin{tabular}{||c|c|cc|cc||}
\hline & $N$ & $L^{2}$ error & order & $L^{\infty}$ error & order \\
\hline \multirow{3}{*}{$\mathcal{P}^{1}$} & 16 & $2.12 \mathrm{E}-02$ & - & $1.52 \mathrm{E}-02$ & - \\
& 32 & $5.33 \mathrm{E}-03$ & 1.99 & $3.85 \mathrm{E}-03$ & 1.99 \\
& 64 & $1.33 \mathrm{E}-03$ & 2.00 & $9.65 \mathrm{E}-04$ & 2.00 \\
\hline \multirow{3}{*}{$\mathcal{P}^{2}$} & 16 & $1.58 \mathrm{E}-03$ & - & $1.31 \mathrm{E}-03$ & - \\
& 32 & $1.85 \mathrm{E}-04$ & 3.09 & $1.63 \mathrm{E}-04$ & 3.00 \\
& 64 & $2.25 \mathrm{E}-05$ & 3.04 & $2.03 \mathrm{E}-05$ & 3.00 \\
\hline
\end{tabular}

To test the temporal accuracy numerically for the second order convex splitting scheme (3.24), we choose $\mathcal{P}^{1}$ elements for spatial discretization and present the $L^{2}$ and $L^{\infty}$ errors and the numerical orders of accuracy at time $T=0.5$ in Table 2 , which shows second order accurate in time.

To demonstrate the near optimal complexity (with respect to the spatial step size $\Delta x$ ) of the multigrid solver, we provide evidence that the multigrid convergence rate is nearly independent of $\Delta x$. We fix the time step as $\Delta t=0.001$ and the spatial step size varies from $\Delta x=2 \pi / 32$ to $\Delta x=2 \pi / 128$. The number of multigrid smoothing sweeps is fixed as 5 . The convergence rates of the multigrid solver at the 10th time step is presented in Fig. 1, we can see the nearly optimal convergence of the solver for $\mathcal{P}^{1}$ and $\mathcal{P}^{2}$ approximations.

To achieve high order accurate in time, we also choose the third order semi-implicit SDC method described in the previous section. Table 3 shows the $L^{2}$ and $L^{\infty}$ errors and the numerical orders of accuracy at time $T=0.5$, which shows $(k+1)$-th order of accuracy for $\mathcal{P}^{k}$ approximation. In Fig. 2, we present numerical solutions at the line $y=x$ with different constant time steps, which shows that our numerical scheme with larger constant time step lose accuracy, i.e., the resolution becomes poorer when the time step becomes larger. 


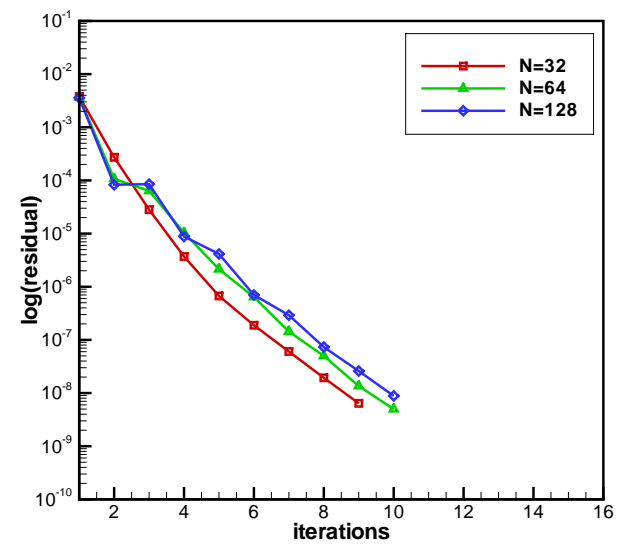

(a) $\mathcal{P}^{1}$

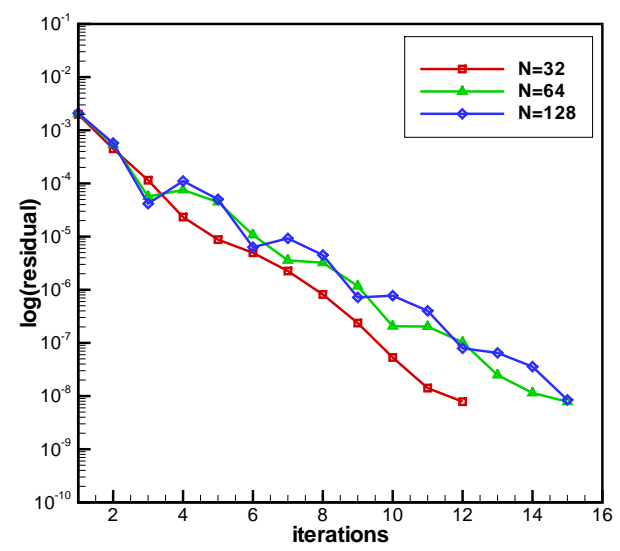

(b) $\mathcal{P}^{2}$

Figure 1: Convergence rates of multigrid solver with $\mathcal{P}^{1}$ and $\mathcal{P}^{2}$ approximation.

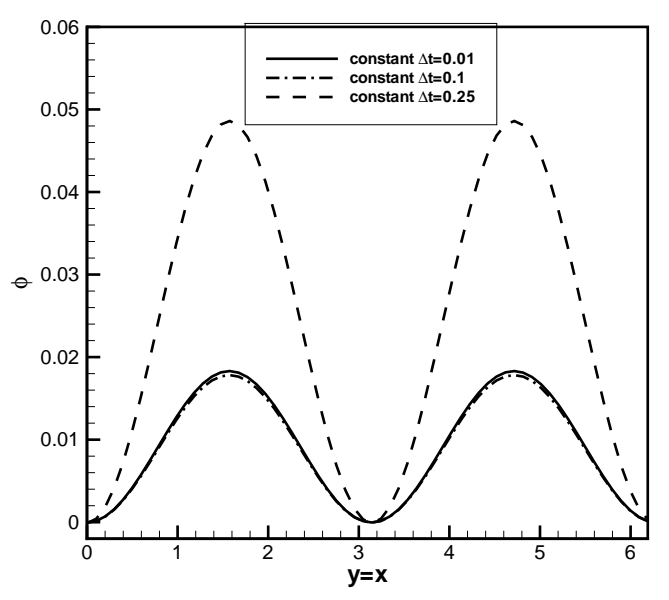

Figure 2: The numerical solution at $y=x$ with different constant time steps for the MPFC equation.

Then, we employ the adaptive time-stepping strategy and study its efficiency. In Fig. 3 (a), we present numerical solutions at the line $y=x$ with adaptive time steps $\Delta t_{1}\left(\Delta t_{\text {min }}=\right.$ $\left.0.01, \Delta t_{\max }=0.1, \alpha=100\right)$ and $\Delta t_{2}\left(\Delta t_{\min }=0.01, \Delta t_{\max }=0.25, \alpha=100\right)$. Obviously, we can see that the numerical accuracy is improved comparing with the results obtained with constant time steps. We also show the comparison of CPU times consumed with constant time step and adaptive time step in Fig. 3 (b). For long time simulation, the adaptive method costs less CPU times, namely, the computational efficiency is improved with adaptive method. 


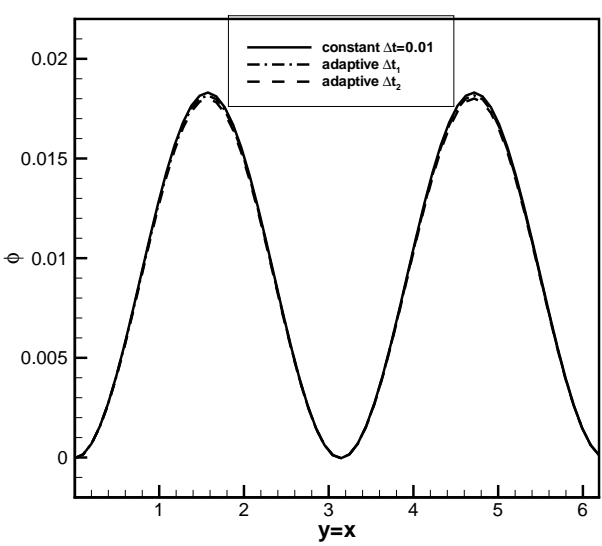

(a) The numerical solution at $y=x$

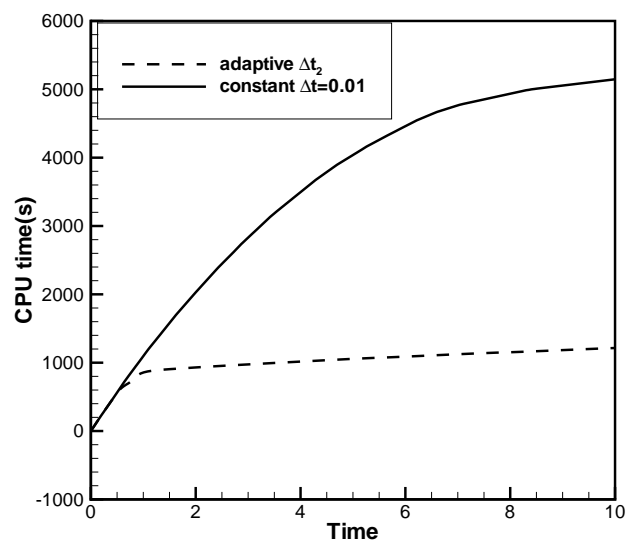

(b) CPU time

Figure 3: (a) The numerical solution at $y=x$ with adaptive time steps $\Delta t_{1}\left(\Delta t_{\min }=0.01, \Delta t_{\max }=0.1, \alpha=100\right)$ and $\Delta t_{2}\left(\Delta t_{\min }=0.01, \Delta t_{\max }=0.25, \alpha=100\right)$. (b) CPU time comparison between constant time step $\Delta t=0.01$ and adaptive time step $\Delta t_{2}$.)

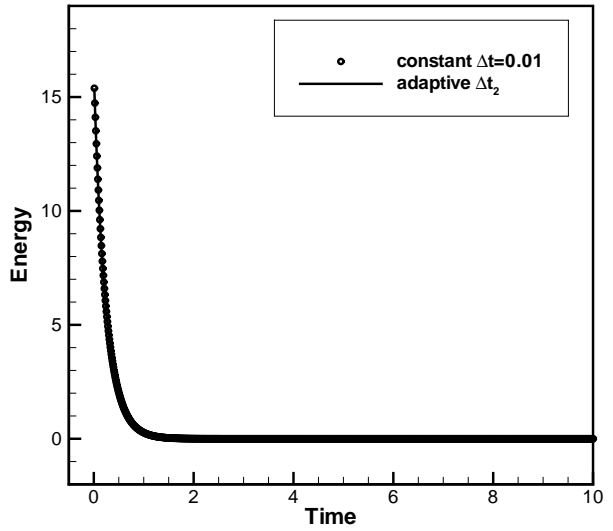

(a) Energy trace

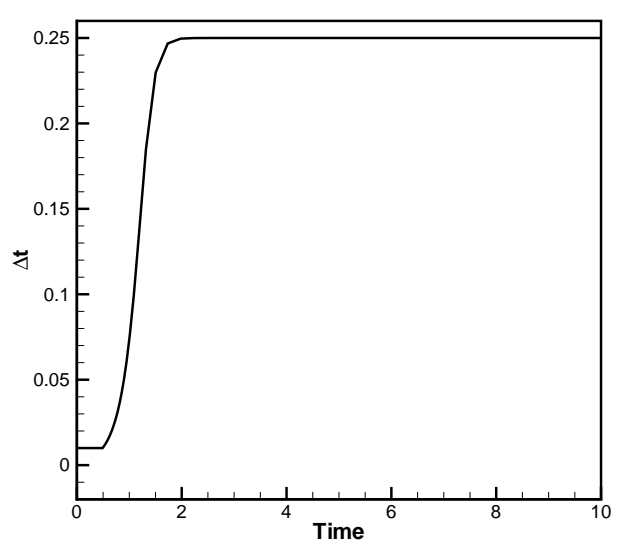

(b) Time step evolution

Figure 4: (a) The discrete energy traces obtained using adaptive time-stepping strategy $\left(\Delta t_{\min }=0.01, \Delta t_{\max }=\right.$ $0.25, \alpha=100)$ and constant time step $(\Delta t=0.01)$. (b) Adaptive time step evolution.

The pseudo energy $\mathcal{E}_{h}$ curves are presented in Fig. 4 (a) obtained using adaptive time step $\Delta t_{2}\left(\Delta t_{\min }=0.01, \Delta t_{\max }=0.25, \alpha=100\right)$ and constant time step $\Delta t=0.01$, respectively. We can see that the two curves coincides, hence the solution dynamics can be captured when an adaptive strategy is employed. In Fig. 4 (b), we also present the corresponding adaptive time steps. 
Example 5.2. (Energy stability test). In this example, the physical parameters are $M=1$, $\epsilon=0.025, \beta=0.01$ and $\Omega=[0,32] \times[0,32]$. The initial condition is taken as

$$
\begin{aligned}
\phi_{0}(x, y)= & 0.07-0.02 \cos \left(\frac{2 \pi(x-12)}{32}\right) \sin \left(\frac{2 \pi(y-1)}{32}\right)+0.02 \cos ^{2}\left(\frac{\pi(x+10)}{32}\right) \\
& \cos ^{2}\left(\frac{\pi(y+3)}{32}\right)-0.01 \sin ^{2}\left(\frac{4 \pi x}{32}\right) \sin ^{2}\left(\frac{4 \pi(y-6)}{32}\right) .
\end{aligned}
$$

To test the energy stability, we use the first order convex splitting scheme (3.2), second order convex splitting scheme (3.24) and the first order linear scheme (2.12) with $\Delta t=0.1$ and advance the evolution to $T=100$. The traces of energy $E_{h}(\phi)$ defined in (2.1), the pseudo energy $\mathcal{E}_{h}(\phi, u)$ defined in (2.6), $\mathcal{F}_{h}$ defined in (3.26) and the energy $\mathcal{E}_{h}(\phi, u, U)$ are presented in Fig. 5. As mentioned above, the energy $E_{h}$ may increase in time on some time intervals, while the pseudo energy $\mathcal{E}_{h}$ and $\mathcal{F}_{h}$ are non-increasing with respect to time. Hence, it is necessary and meaningful to introduce the pseudo energy.

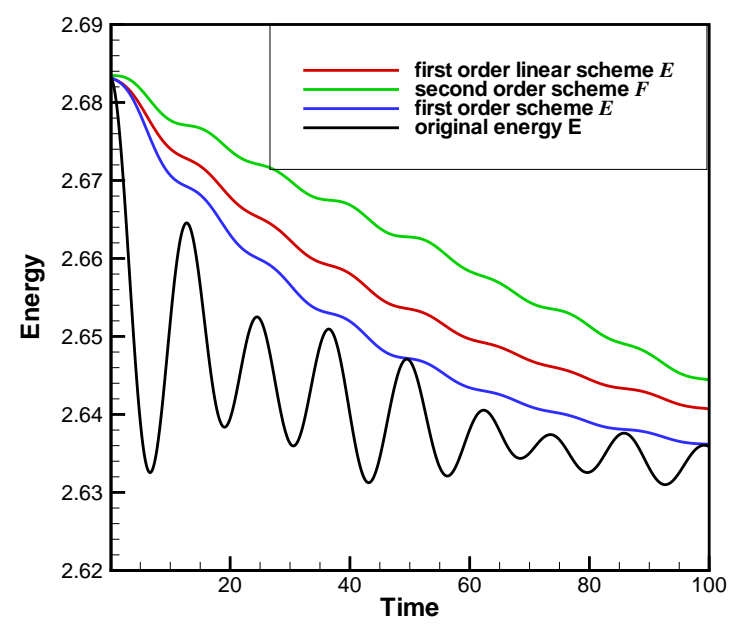

Figure 5: The computed discrete energies $E_{h}(\phi)$ (black), $\mathcal{E}_{h}(\phi, u)$ (blue), $\mathcal{F}_{h}$ (green) and $\mathcal{E}_{h}(\phi, u, U)($ red) plotted as functions of time.

Example 5.3. (Long time simulation). In this example, we will show the long time characteristic of the MPFC model with the random initial data on a square domain $[0,128] \times$ $[0,128]$. The initial data takes $\phi_{i, j}^{0}=\bar{\phi}+\eta_{i, j}$, where $\bar{\phi}=0.07$ and $\eta_{i, j}$ is an uniformly distributed random number satisfying $\left|\eta_{i, j}\right| \leq 0.07$. The parameters are $M=1, \beta=0.9$ and $\epsilon=0.025$.

The computational parameters are the spatial discretization cell size $h=128 / \mathrm{N}$ with $N=128$ and the piecewise $\mathcal{P}^{2}$ approximation. We employ the third order semi-implicit SDC method, and the adaptive time step defined in (4.4) with $\alpha=10^{5}$. Numerical results 


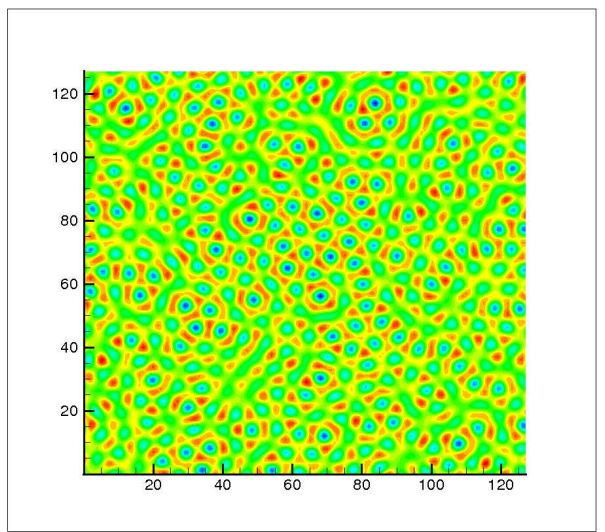

(a) $T=400$, adaptive $\Delta t_{1}$

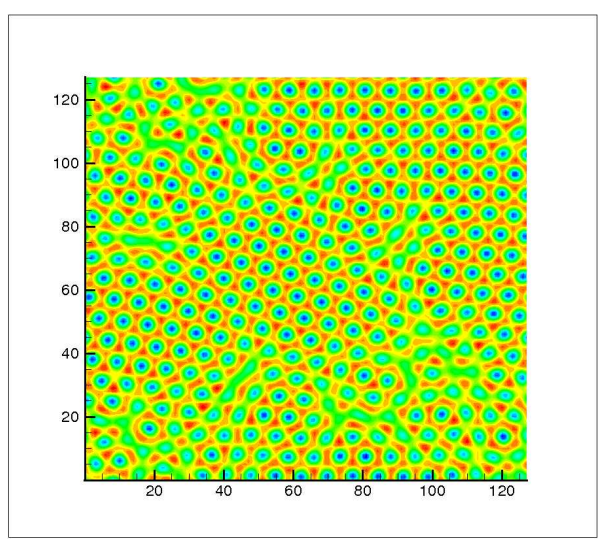

(c) $T=1500$, adaptive $\Delta t_{2}$

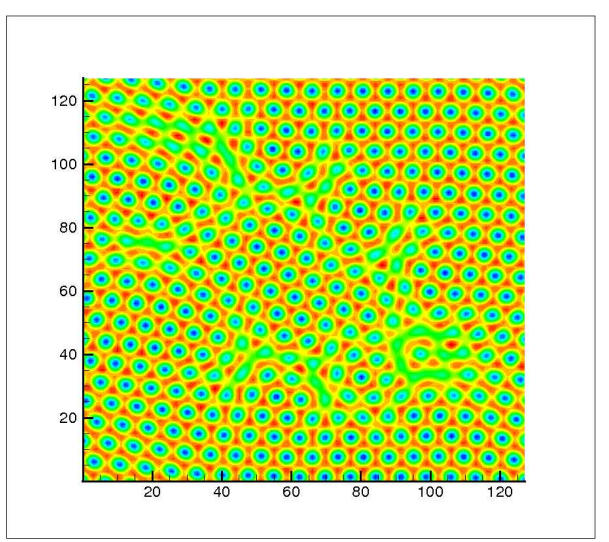

(e) $T=5000$, adaptive $\Delta t_{3}$

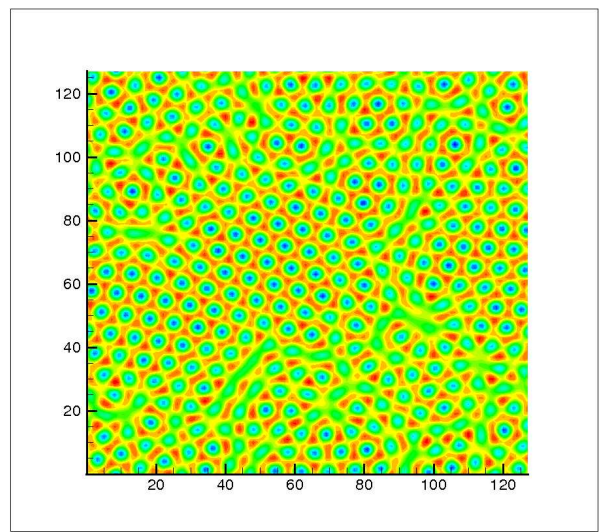

(b) $T=800$, adaptive $\Delta t_{1}$

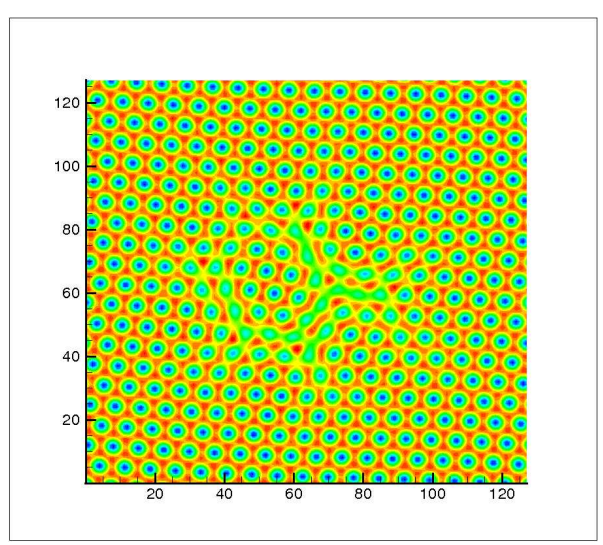

(d) $T=3000$, adaptive $\Delta t_{2}$

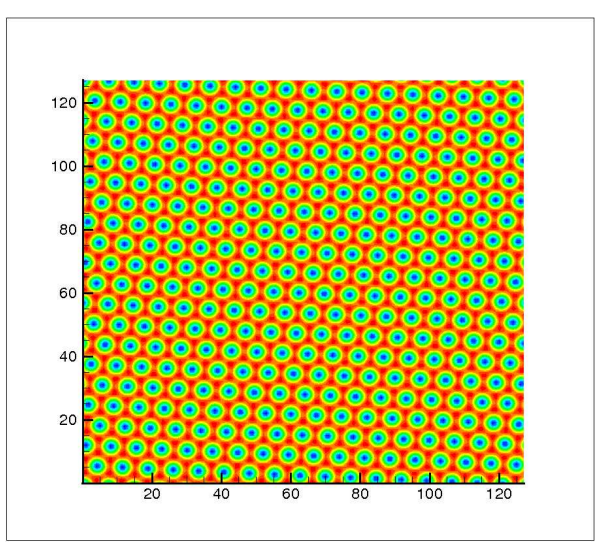

(f) $T=10000$, adaptive $\Delta t_{3}$

Figure 6: Numerical solutions of the MPFC equation obtained using the third order SDC method with adaptive time-stepping strategy $\Delta t_{1}, \Delta t_{2}$ and $\Delta t_{3}$. 


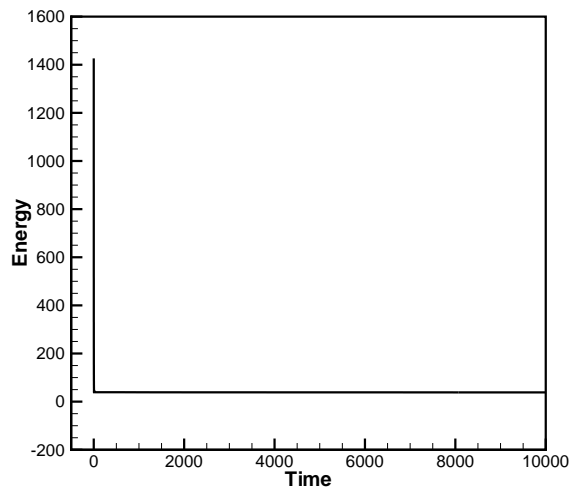

(a) Energy trace from $T=0$ to $T=10000$

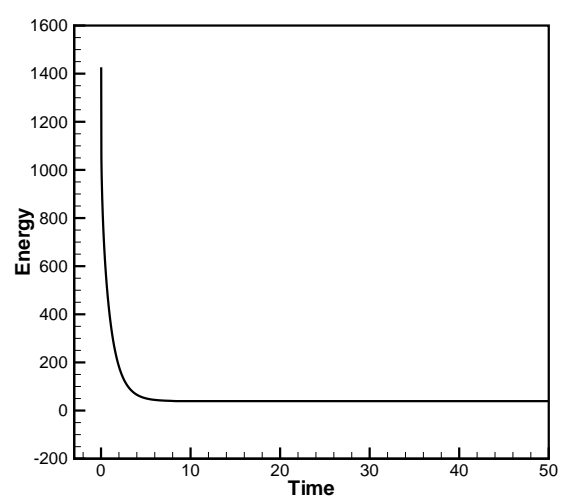

(c) Energy trace from $T=0$ to $T=50$

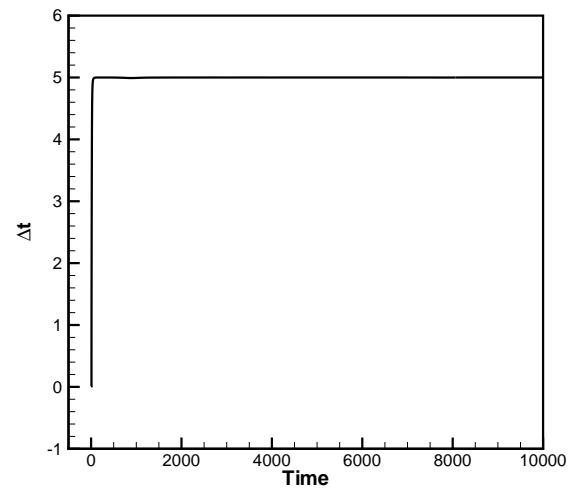

(b) Time step from $T=0$ to $T=10000$

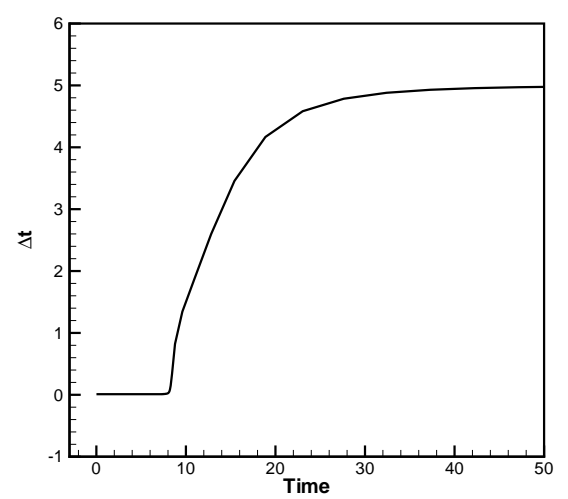

(d) Time step from $T=0$ to $T=50$

Figure 7: $(\mathrm{a})(\mathrm{c})$ The discrete energy traces of the MPFC equation using the adaptive time-stepping strategy $\left(\Delta t_{\min }=0.01, \Delta t_{\max }=5.0, \alpha=10^{5}\right)$. (b)(d) The adaptive time steps.

using different adaptive time steps $\Delta t_{1}\left(\Delta t_{\min }=0.01, \Delta t_{\max }=0.1\right), \Delta t_{2}\left(\Delta t_{\min }=0.01, \Delta t_{\max }=\right.$ 1.0) and $\Delta t_{3}\left(\Delta t_{\min }=0.01, \Delta t_{\max }=5.0\right)$ are presented in Fig. 6. It is observed that the solution dynamics can be captured correctly with adaptive time-stepping strategy.

The energy curve is presented in Fig. 7 (a), it decays quickly at the early stage and then the energy decays rather slowly. The corresponding time step are given in Fig. 7 (b), which agrees with the energy evolution. To show the energy trace and time step more clearly, we zone them from $T=0$ to $T=50$ and show the results in Figs. 7 (c) and 7 (d), respectively.

Example 5.4. (Growth of a polycrystal). The last example presents the growth of a polycrystal in a supercooled liquid. To define the initial configuration, we proceed as follows: 
first setting all control variables to a constant value $\bar{\phi}=0.285$, and then modifying this constant configuration by setting three perfect crystallites in three small square patches of the domain. The crystallites are defined as follows:

$$
\phi\left(x_{l}, y_{l}\right)=\bar{\phi}+C\left[\cos \left(\frac{q}{\sqrt{3}} y_{l}\right) \cos \left(q x_{l}\right)-0.5 \cos \left(\frac{2 q}{\sqrt{3}} y_{l}\right)\right],
$$

where $x_{l}$ and $y_{l}$ define a local system of cartesian coordinates that is oriented with the crystallite lattice. Here, the local coordinates $\left(x_{l}, y_{l}\right)$ are defined by an affine transformation of the global coordinates $(x, y)$, which produces a rotation given by an angle $\theta$. Also, $\theta$ are chosen as $-\frac{\pi}{4}, 0$, and $\frac{\pi}{4}$, respectively, to generate crystallite lattices with different orientations. We take the parameter $C=0.446$ and $q=0.66$.

The square domain is $(0,804) \times(0,804)$ with $\epsilon=0.25$ and $M(\phi)=1$. The boundary conditions are periodic in the horizontal direction and homogeneous Neumann on the top and bottom boundaries. The computational parameters are the spatial discretization cell size $\Delta x=804 / N$ with $N=512$ and the piecewise $\mathcal{P}^{k}$ polynomials basis with $k=1$. For time discretization, we employ the third order semi-implicit SDC method, and the adaptive time step defined in (4.4) with $\Delta t_{\min }=0.1, \Delta t_{\max }=1.0, \alpha=100$.

In order to study the effect of the damping term, we take $\beta=0.9$ and $\beta=10$, respectively, to simulate our numerical solutions, with the same initial data and parameters. Numerical results at different times obtained with $\beta=0.9$ are presented in Fig. 8, while for $\beta=10$, the numerical result at time $T=3000$ is shown in Fig. 10. All these results show statistically similar patterns in the numerical solutions as those in [1].

Fig. 9 (a) shows the discrete energy trace of the numerical solution, We can see that the energy is non-increasing in time, which agrees with the theoretical result. In addition, the energy decays quickly at the early stage and then it decays rather slowly. The corresponding time step is given in Fig. 9 (b), which agrees with the energy evolution.

\section{Concluding remarks}

In this paper, we developed several energy stable local discontinuous Galerkin schemes for the modified phase field crystal equation, and proved the corresponding unconditional energy stabilities and the boundness of the numerical solution. What made it different and difficult comparing with the numerical scheme for phase field crystal equation was the treatment of the second-order time derivative $\partial_{t t} \phi$ and the introduction of pseudo energy. The most important property of LDG methods is high order accurate, and in order to achieve higher order temporal accuracy, the semi-implicit spectral deferred correction method was employed, combining with the first order convex splitting scheme. The numerical simulations of modified phase field crystal model always need long time to reach steady state, and therefore adaptive time-stepping strategy was adopted. Numerical experiments were presented to show that the proposed schemes were indeed high order accurate in time and space. In addition, long time simulations were presented to 


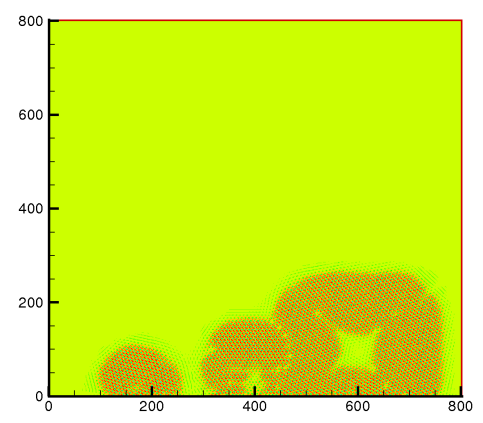

(a) $T=200$

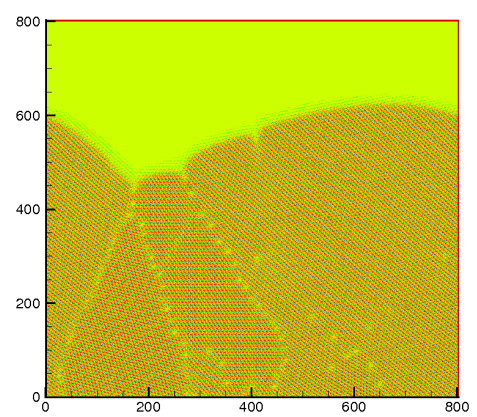

(c) $T=1000$

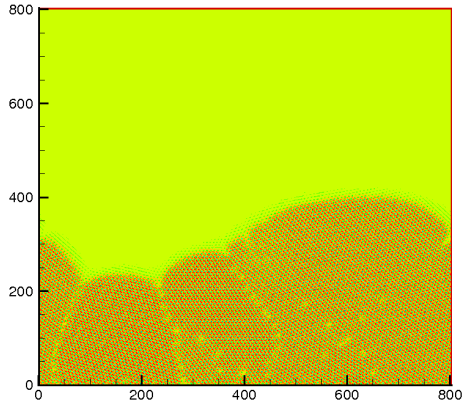

(b) $T=500$

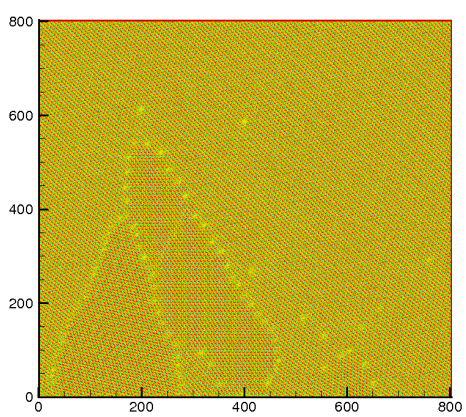

(d) $T=2000$

Figure 8: $\beta=0.9$ : Numerical solutions of the MPFC equation obtained using the third order SDC method with adaptive time step sizes.

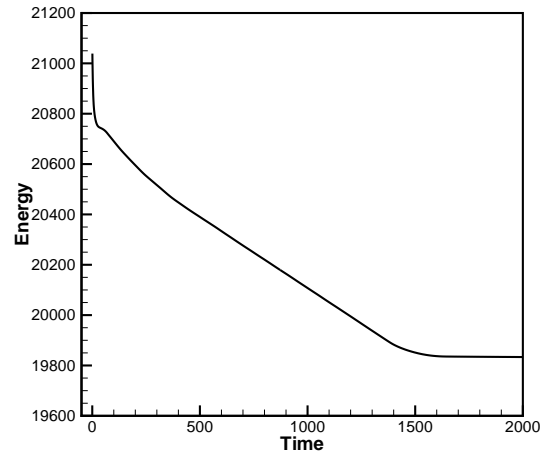

(a) Energy trace

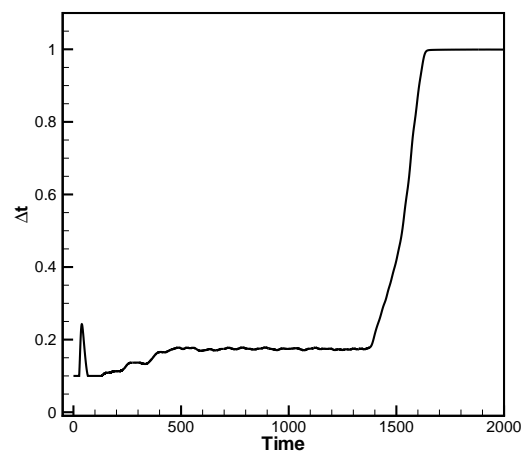

(b) Time step

Figure 9: $\beta=0.9$ : (a) The discrete energy traces of the MPFC equation obtained using the adaptive time-stepping strategy $\left(\Delta t_{\min }=0.1, \Delta t_{\max }=1.0, \alpha=100\right)$. (b) The adaptive time step evolutions. 


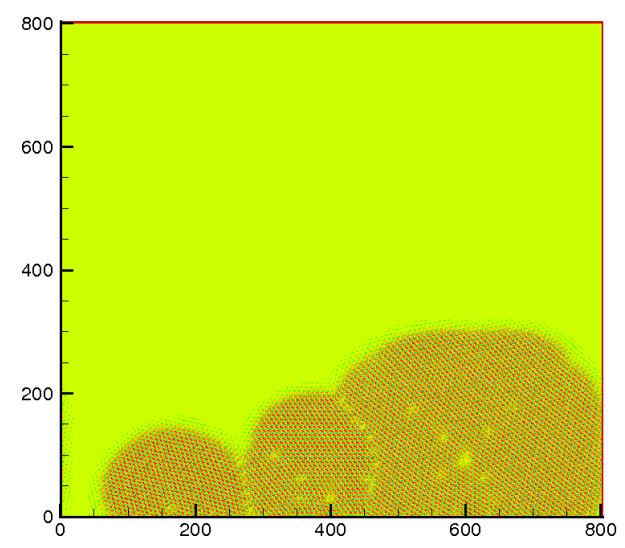

Figure 10: $\beta=10$ : Numerical solutions of the MPFC equation obtained using the third order SDC method with adaptive time step sizes.

demonstrate the capability and efficiency of our proposed approaches when solving the modified phase field crystal equation.

\section{Acknowledgments}

Research of R. Guo is supported by NSFC grant No. 11601490. Research of Y. Xu is supported by NSFC grant No. 11371342, 11626253, 91630207.

\section{References}

[1] A. Baskaran, Z. Hu, J.S. Lowengrub, C. Wang, S.M. Wise and P. Zhou, Energy stable and efficient finite-difference nonlinear-multigrid schemes for the modified phase field crystal equation, J. Comput. Phys., 250 (2013), pp. 270-292.

[2] A. Baskaran, J.S. Lowengrub, C. Wang and S.M. Wise, Convergence analysis of a second order convex splitting scheme for the modified phase field crystal equation, SIAM J. Numer. Anal., 51 (2013), pp. 270-292.

[3] F. Bassi and S. Rebay, A high-order accurate discontinuous finite element method for the numerical solution of the compressible Navier-Stokes equations, J. Comput. Phys., 131 (1997), pp. 267-279.

[4] A. Brandt, Multigrid techniques: 1984 guide with applications to fluid dynamics, GMD-Studien [GMD Studies], 85. Gesellschaft für Mathematik und Datenverarbeitung mbH, St. Augustin, 1984.

[5] B. Cockburn and C.-W. Shu, TVB Runge-Kutta local projection discontinuous Galerkin finite element method for conservation laws II: general framework, Math. Comp., 52 (1989), pp. 411-435.

[6] B. Cockburn, S.-Y. Lin and C.-W. Shu, TVB Runge-Kutta local projection discontinuous Galerkin finite element method for conservation laws III: one dimensional systems, J. Comput. Phys., 84 (1989), pp. 90-113. 
[7] B. Cockburn, S. Hou and C.-W. Shu, The Runge-Kutta local projection discontinuous Galerkin finite element method for conservation laws IV: the multidimensional case, Math. Comp., 54 (1990), pp. 545-581.

[8] B. Cockburn and C.-W. Shu, The Runge-Kutta discontinuous Galerkin method for conservation laws V: multidimensional systems, J. Comput. Phys., 141 (1998), pp. 199-224.

[9] B. Cockburn and C.-W. Shu, The local discontinuous Galerkin method for time-dependent convection-diffusion systems, SIAM J. Numer. Anal., 35, (1998), pp. 2440-2463.

[10] K.R. Elder, M. Katakowski, M. Haataja and M. Grant, Modeling elasticity in crystal growth, Phys. Rev. Lett., 88 (2002), article 245701.

[11] K.R. Elder and M. Grant, Modeling elastic and plastic deformations in nonequilibrium processing using phase field crystal, Phys. Rev. E, 68 (2003), pp. 066703.

[12] P.K. Galenko, H. Gomez, N.V. Kropotion and K.R. Elder, Unconditionally stable method and numerical solution of the hyperbolic phase-field crystal equation, Physical Review E, 88 (2013), pp. 013310.

[13] H. Gomez and X. Noguerira, An unconditionally energy-stable method for the phase field crystal equation, Comput. Methods Appl. Mech. Engrg., 249 (2012), pp. 52-61.

[14] M. Grasselli and M. Pierre, Energy stable and convergent finite element schemes for the modified phase field crystal equation, Forestry Chronicle, 87 (2015), pp. 755-765.

[15] R. Guo and Y. Xu, Local discontinuous Galerkin method and high order semi-implicit scheme for the phase field crystal equation, SIAM J. Sci. Comput., 38 (2016), pp. A105-A127.

[16] R. Guo, Y. Xia and Y. Xu, Semi-implicit spectral deferred correction methods for highly nonlinear partial differential equations, J. Comput. Phys., 338 (2017), pp. 269-284.

[17] Z. Hu, S.M. Wise, C. Wang and J.S. Lowengrub, Stable and efficient finite-difference nonlinearmultigrid schemes for the phase field crystal equation, J. Comput. Phys., 228 (2009), pp. 5323-5339.

[18] W.H. Reed and T.R. Hill, Triangular mesh method for the neutron transport equation, Technical report LA-UR-73-479, Los Alamos Scientific Laboratory, Los Alamos, NM, 1973.

[19] H. Song and C.-W. Shu, Unconditionally energy stability analysis of a second order implicit-explicit local discontinuous Galerkin method for the Cahn-Hilliard equation, J. of Sci. Comput., 73 (2017), pp. 1178-1203.

[20] P. Stefanovic, M. Haataja and N. Provatas, Phase-field crystals with elastic interactions, Phys. Rev. Lett., 96 (2006), article 225504.

[21] P. Stefanovic, M. Haataja and N. Provatas, Phase field crystal study of deformation and plasticity in nanocrystalline materials, Phys. Rev. E, 80 (2009), article 046107.

[22] C. Wang and S. Wise, An energy stable and convergent finite-difference scheme for the modified phase field crystal equation, SIAM J. Numer. Anal., 49 (2011), pp. 945-969.

[23] S.M. Wise, C. Wang and J.S. Lowengrub, An energy-stable and convergent finite-difference scheme for the phase field crystal equation, SIAM J. Numer. Anal., 44 (2009), pp. 2269-2288.

[24] Y. Xia, Y. Xu and C.-W. Shu, Efficient time discretization for local discontinuous Galerkin methods, Discrete Contin. Dyn. Syst., Ser. A 8 (2007), pp. 677-693.

[25] Y. Xu and C.-W. Shu, Local discontinuous Galerkin methods for high-order time-dependent partial differential equations, Commun. Comput. Phys., 7, (2010), pp. 1-46.

[26] X. Yang and D. Han, Linearly first-and second-order, unconditionally energy stable schemes for the phase field crystal model, J. Comput. Phys., 330 (2017), pp. 1116-1134.

[27] Z. Zhang, Y. Ma and Z. Qiao, An adaptive time-stepping strategy for solving the phase field crystal model, J. Comput. Phys., 249 (2013), pp. 204-215. 